\title{
INTEGRATION OF PARTS SCHEDULING, MRP, PRODUCTION PLANNING AND GENERALIZED FIXED-CHARGE TRANSPORTATION PLANNING IN THE DESIGN OF A DYNAMIC CELLULAR MANUFACTURING SYSTEM
}

\author{
Shima Shafiee-Gol ${ }^{1}$, Reza Kia ${ }^{2,3, *}$, Reza Tavakkoli-Moghaddam ${ }^{1}$, \\ Mohammad Kazemi ${ }^{4}$ And Mehdi A. Kamran ${ }^{2,5}$
}

\begin{abstract}
In this paper, to integrate the decisions of parts scheduling, Material Requirement Planning (MRP), Production Planning (PP) and Transportation Planning (TP) for designing a Cellular Manufacturing System (CMS) under a dynamic environment, a Mixed-Integer Nonlinear Programming (MINLP) mathematical model is formulated. The proposed mathematical model integrates extensive coverage of significant manufacturing characteristics in designing a CMS to be implemented in a threelayer supply chain. The considered features include markets demands, heterogeneous vehicles, raw materials requirements planning, parts due dates, cell size limits, machines capacity, intra/inter cell material handling time/cost, transportation time/cost, operation time, alternative processing routes in addition to the main decisions of parts scheduling, PP, TP and dynamic cell formation. Also, some novel characteristics are incorporated based on a three-layer supply chain that make the presented model remarkable respect to the literature including (1) In the first layer, planning the orders of raw materials with different lead times and usage coefficients is performed, (2) In the second layer, decisions of dynamic cell formation and parts scheduling are made, and (3) In the third layer, optimal vehicles are selected as a generalized fixed-charge TP based on transportation time and cost to satisfy multimarkets with different demand volumes. The components in the objective function to be minimized include total costs of holding the parts inventories in the markets, backorders, tardiness, transportation of the parts from the plant to the markets, purchase of raw materials, keeping raw materials in the plant warehouse, intercellular/intracellular movements and machine relocation. An illustrative numerical example is solved by the CPLEX solver to illustrate the achievements obtained by the incorporated characteristics in the integrated model. Furthermore, a sensitivity analysis is performed to assess the effects of important parameters on the model performance. Since the proposed model is NP-hard, a Simulated Annealing (SA) algorithm is improved by an elaborately-designed matrix-based chromosome representation is applied to represent all decision variables, as well as a sequential procedure generating initial solutions. Several test problems either generated randomly or taken from the literature with various sizes are solved and the results are compared with the solutions gained using CPLEX solver.
\end{abstract}

Keywords. Dynamic cellular manufacturing system, three-layer supply chain, scheduling, production planning, transportation planning.

1 School of Industrial Engineering, College of Engineering, University of Tehran, Tehran, Iran.

2 Department of Logistics, Tourism, \& Service Management/Faculty of Business \& Economics, German University of Technology (GUtech), Muscat, Oman.

3 Department of Industrial Engineering, Firoozkooh Branch, Islamic Azad University, Firoozkooh, Iran.

4 Department of Industrial Engineering, Birjand University of Technology, Birjand, Iran.

5 Department of Industrial Engineering, Urmia University of Technology, Urmia, Iran.

*Corresponding author: reza.kia@gutech.edu.om 
The comparisons results show that the designed SA is capable of evolving optimal or near-optimal solutions with reasonable relative gaps in a computationally satisfactory manner.

Mathematics Subject Classification. 90-10.

Received November 9, 2017. Accepted June 11, 2020.

\section{INTRODUCTION}

Nowadays, a business needs to concentrate on needs and interests of demanding products of customers with both higher quality and lower price to survive in the competitive business world. Therefore, many businesses try to acquire the ability of agility and fast-responding to the customers' demands. To achieve this goal, Cellular Manufacturing (CM) can be employed as an industrial application of Group Technology (GT). It was introduced by Mitrofanov [38] and extended by Burbidge [9]. The significant benefits of implanting CM are decreasing in setup time, flow-time, Work-in-Process (WIP) level, material handling costs, improvement in quality and production supervision, and increase in flexibility, etc. [23,61].

Often fluctuations occurring in product demands and product mixes lead to a dynamic manufacturing environment due to increasing the variety of customers' products and decreasing the life cycles of products. In a dynamic environment, where the product mixes and part demands change during a multi-period planning horizon, it is necessary to reconfigure cells efficiently for consecutive periods. This type of CM was presented as the Dynamic Cellular Manufacturing System (DCMS) by Rheault et al. [53]. It was indicated by Drolet et al. [15] that DCMSs are normally more efficient than traditional CMSs and job shops through developing a simulation model, especially with regarding some performance measures such as flow-time, WIP, tardiness and the total costs for a known planning horizon. Afterwards, some mathematical models and solution procedures have been proposed by several researchers considering DCMSs under multiple periods $[7,8,26,27]$. In these studies, it was assumed that the production amount of each part equals demand volume in each period.

In practice, production quantity may not be equal to the demand volume since it could be also fulfilled through other strategies such as holding inventory, backorders or outsourcing. Hence, production quantity should be decided using Production Planning (PP) decisions including internal production, inventory, backorder or outsourcing. Afterwards, the number and type of machines to be utilized in the system could be determined. Some papers have incorporated the aforementioned decisions in DCMS [3,12,48].

In our model, the demand of a market in a period has to be satisfied in one of the periods that may be one or a few periods earlier than due date using inventory holding, one or a few periods later than the due date as backorders and or in the same period using the internal production.

The design of a CMS in a plant that is a manufacturer in a supply chain involves various structural and operational decisions [60]. The major structural decisions are grouping parts and machines into cells (i.e., Cell Formation (CF) problem; and cells reconfiguration during successive periods); and operational ones are production planning and scheduling; as well transportation planning from the plant to markets. The impact of integrating these decisions on the CMS design and its performance is studied by various researchers in different focus with different objectives. In manufacturing environments, these decisions are inter-linked. Therefore, the need arises to develop models considering them as an integrated whole. Indeed, it cannot be guaranteed that optimal decisions obtained from a problem that only models with one of the aforementioned decisions will be optimal for another problem separately. Indeed, a real industrial environment comprises all of these decisions together. For instance, the obtained solutions satisfying only limitations of the CF problem cannot satisfy the limitations of a problem integrating all factors. Then, the final design may not be efficient and optimal. Consequently, to have a model whose decisions are comparable with practical decisions, a comprehensive model is necessary.

Rao and Mohanty [49] explored the interrelationships between CMS and Supply Chain (SC) design issues. Simultaneous decision making of SC and CM concepts results in incurring lower costs of distribution and 
production and responding to customers more rapidly. Recently, the necessity of integration between DCMS and SCM decisions has been studied through mathematical models $[1,36,45]$. In the traditional Transportation Problem (TP) as a basic, practical and famous optimization problem in operations research, there are two types of constraints, namely supply constraint and demand constraint and the decision variable to be obtained is how many items should be transported from each source to each -*/destination [40]. The generalized TP where there is more than one type of products to be transported between plants and markets is a basis in designing a supply chain network with several layers. Also, in practical situations some problems may consist of fixed costs which are independent of the quantity of transported load due to tax charges and opening routes. In our study, the layer between the plant and markets is considered as a Generalized Fixed-Charge Transportation Problem (GFCTP).

In addition to the CF step in designing a CMS that includes clustering parts with similar manufacturing necessities into the part families and matching machines to cells, another major step is Group Scheduling (GS) (i.e., scheduling of part families) [62]. The necessity for integration of CF and GS decisions has been highlighted by some mathematical models $[6,16,58]$.

In our model, a three-layer SC is addressed including: (1) the first layer plans the orders of raw materials with different lead times and usage coefficients, (2) the second layer determines dynamic cell formation and parts scheduling, and (3) the third layer selects the optimal transportation vehicle for each part produced in the plant to be transported to each market as a transportation problem. In fact, in the first layer, an MRP is devised to determine the launching times and quantities of orders for raw materials to be assembled in final products with one-level Bill-Of-Materials (BOM). In the second layer (i.e., shop floor), parts scheduling determines the starting and finishing times of each part operation, the completion time of each part and the amount of tardiness for each part based on the deviation between the part due date and its delivering time to a market. Furthermore, in this layer, cell formation decisions in a dynamic environment are taken to determine the allocation of machines to cells in each period as well as the relocation of machines among cells during successive periods due to required manufacturing capacity changing based on fluctuating demands. Finally, in the third layer, selecting heterogeneous vehicles with different speeds for responding to multi-markets with different demand volumes is made as a GFCTP.

The complexities associated with the NP-hardness and large search space of the model demand the usage of an intelligent search heuristic method, which works with a simultaneous set of solutions in parallel to avoid search ending in poor quality solutions and directs the search to a global or near-optimal solution. Many intelligent search meta-heuristics (e.g., Simulated Annealing (SA), Tabu Search (TS), Genetic algorithm (GA), Bacteria Foraging Algorithm (BFA)) have been proposed for many CMS design problems. However, SA has found a special place in CMS studies due to its strength in handling complex problems, especially with numerous constraints that are common in CMS models. Therefore, many studies have recently employed SA as an efficient solution approach and reported satisfactory results $[8,12,17,21,24,33,51,56,64]$

The main contribution of this paper, regarding the reviewed articles and unveiled necessities of integrating the discussed decisions including $\mathrm{CF}$, scheduling, TP and PP is presenting a comprehensive model for designing a DCMS into an SC integrating (1) CF under a dynamic environment, (2) MRP with one-level BOM, (3) parts scheduling, (4) GFCTP and (5) PP strategies for satisfying multi-markets demands. The integrated model simultaneously incorporates several important design features including multi-markets with different parts demands and due dates, heterogeneous vehicles with different speed and fixed charges, raw materials with different lead times and usage coefficient, cell size limits, machines capacity, material handling time, operation time, alternative processing routes, employing inventory holding and backorder strategies for fulfilling markets demands, keeping inventories of raw materials in the plant warehouse, cell reconfiguration, intra/inter-cellular movement and operations sequence to bring the model closer to manufacturing realities.

To the best of our knowledge, this is the first time that a mathematical model integrating the decisions of MRP, scheduling, GFCTP and PP to configure a CMS into an SC structure under a dynamic environment is formulated with comprehensive incorporation of important design features in the model objective function and constraints. 
Another contribution of this paper is designing an efficient SA algorithm boosted by a matrix-based chromosome representation involving three matrices to represent all decision variables and a hierarchical procedure generating initial feasible solutions to determine the interrelated decisions of MRP, scheduling, GFCTP and PP in designing a DCMS simultaneously.

A demonstrative numerical example is explained by the CPLEX solver to assess the effects of simultaneous integration of mentioned decisions along with other incorporated design features in designing a DCMS. Additionally, to verify the efficiency of the developed SA, several numerical examples are solved and its results are compared to those obtained for the examples solved by CPLEX solver. The outcomes reveal the effectiveness of SA in attaining near-optimal solutions in reasonable computational times.

The remainder of this paper is ordered as follows. A literature review related to CM design integrated with $\mathrm{PP}$, scheduling and supply chain is given in Section 2. A mathematical model integrating decisions of MRP, scheduling, GFCTP and PP in designing a DCMS is presented in Section 3. The development of the designed $\mathrm{SA}$ is discussed in Section 4. An illustrative example is presented and solved using CPLEX solver in Section 5. Also, the test problems are solved to investigate the features of the model, sensitivity analysis and assessing the performance of the designed SA. In the end, Section 6 draws conclusions and future studies directions.

\section{Literature REVIEW}

Since the presented model integrates different decision areas including parts scheduling, production planning and transportation planning in designing a CMS, some recent papers addressed the integration of at least one of the mentioned areas in CMS are reviewed. Furthermore, some studies developed a SA-based solution approach for designing a CMS are considered.

\subsection{CMS studies integrated with SC or TP}

Olhager [43] took a historical outlook recognizing the main trends and application shifts in the development of planning and control, from the shop floor control through MRP, Master Production Schedule (MPS), and Sales and Operations Planning (S\&OP) to SCM. Ghezavati et al. [20] studied a robust mathematical model for a CF problem integrated with features of an SC network considering uncertain manufacturing times and demands. The objective was to minimize the expected value and variance of total costs including the scheduling cost, employment cost of suppliers in SC network, manufacturing exceptional elements in addition to the resource underutilization cost. Lokesh and Jain [36] presented an integrated model of the DCMS and SC design considering several issues including multi-plant locations, multiple markets, reconfiguration, etc. Paydar et al. [46] developed a robust optimization model to solve a mathematical model integrating purchase and PP in the design of an SC and CMS, simultaneously with uncertain demands and machine capacities. In another similar study, Paydar and Saidi-Mehrabad [45] developed a Multi-Choice Goal Programming (MCGP) method to solve a bi-objective possibilistic model to combine procurement, production and distribution planning with the simultaneous consideration of several conflicting objectives along with the rough nature of several significant parameters in designing a dynamic VCMS. Aalaei and Davoudpour [1] formulated a mathematical model for a CMS into the SC design with a worker assignment and considered important manufacturing features including multiple-plant locations, multi-markets allocations, production planning and numerous part mix. The objective function was the total cost of inventories holding, inter-cellular material handling, external transportation, fixed cost for manufacturing, machine and workers' salaries. Liu et al. [35] proposed a novel optimization model of the DCMS in a supply chain and developed an integrated bacteria foraging algorithm. The advantage of the proposed model was concurrently dealing with facility transfer and production planning by assuming multiple factories and multi-skilled workers.

\subsection{CMS studies integrated with PP}

Khaksar-Haghani et al. [25] formulated an MINLP model for designing a DCMS by incorporation of PP decisions. The total costs of cell establishment, cell underutilization, part-operations setup and manufacturing, 
outsourcing, backorders, inventories holding, material handling between CMS and the warehouse, inter and intra-cell movements and machine relocation were the components of the objective function. Aghajani et al. [2] formulated a mathematical model for designing a DCMS with considering alternative processing routes, lot splitting feature, operations sequence, duplicates of machines, the capacity of machines, manufacturing cost, setup cost, cell size restrictions, and PP decisions. Kia et al. [28] presented an MINLP model to design a GL of a DCMS and consider production planning (PP) decisions, the variable number of cells, and machine depot. Li et al. [31] proposed a production planning algorithm in virtual cells for reconfigurable manufacturing system with unstable production batches. Using an improved GA, the cost from scheduling processes and cell formation considered in the objective function was minimized. Deep and Singh [11] formulated a mathematical model for designing a robust CMS with dynamic part production. They incorporated cell configuration problem with the machines allocation, PP and the part routing. Ghezavati [19] integrated both the CF decision and aspects of production planning into a single mathematical model to design a DCMS, in which stochastic demand has been described by a discrete set of scenarios. Alhourani [5] developed a new similarity coefficient equation that incorporates several production factors including machines reliability, operations sequence, production volumes as well as machines capacity and machine duplicates in the presence of the part route selection to obtain better machine grouping and minimum total cost in designing a CMS. Sakhaii et al. [55] developed a robust optimization approach for an integrated mixed-integer linear programming model to solve a DCMS with unreliable machines and a production planning problem simultaneously. Liu et al. [34] formulated an optimization model of DCMS and designed a hybrid bacteria foraging algorithm embedding two-phase based heuristic to minimize the sum of backorder cost and holding cost of inventory. The proposed model simultaneously considered dynamic multiskilled worker assignment/reassignment, production planning, learning and forgetting effects and operation sequence. Raoofpanah et al. [50] formulated a mathematical model for designing DCMSs with supplier selection, in which environmental issues, inventory balance and production planning are incorporated. Also, a robust optimization model was proposed to handle uncertainty in part processing times.

\subsection{CMS studies integrated with scheduling}

Ghezavati [18] integrated the design of a CMS with GS and GL with uncertain parameters under supply chain characteristics to optimize expected holding cost and the suppliers' network-related costs, in which suppliers are demanded to manufacture exceptional products. Pajoutan et al. [44] developed a SA-based heuristic to solve a mathematical model for a GS problem considering movement time and flexible routing. To minimize the total tardiness penalty cost, Tang et al. [59] employed a Lagrangian relaxation decomposition method to provide an effective solution for the integrated decisions of CF and GS. Alfieri and Nicosia [4] studied the parts' operations scheduling problem in flexible CMSs where the manufacturing cell with a single machine can accommodate up to $k$ parts simultaneously. A sequence of different tools is required for processing of each part that enforces a sequence of tools changes. Therefore, the objective is to minimize the total number of setups with constant times for switching tools. Halat and Bashirzadeh [22] presented a simultaneous approach for the operations scheduling problem in a CMS to minimize the makespan. They developed an MILP model by considering exceptional elements, inter-cell movements, inter-cell transportation times, and sequence-dependent setup times of part families. Li et al. [32] developed a hybrid harmony search for solving a flow-line scheduling problem in a CMS having part families with sequence-dependent setup times to minimize total tardiness and mean total flow-time. Renna and Ambrico [52] addressed reconfigurable machines to design a CMS with changing demand conditions. To incorporate the design, reconfiguration and scheduling of the CMS, an approach based on three mathematical models was proposed.

Delgoshaei [14] proposed a short-term period scheduling method for small and medium scale DCMSs considering bottleneck and parallel machines to find the best production strategy of internal manufacturing and outsourcing. Delgoshaei et al. [13] examined the impact of inflation on cell-load variation in CMSs by proposing a new method for scheduling DCMS in the presence of bottleneck and parallel machines. They developed a hybrid GA and SA algorithm. Ebrahimi et al. [16] presented an MINLP model for designing a CMS to simultaneously integrate the interrelated decisions of machine layout and part scheduling and to combine several design features, 
such as parts due date, movement time, operation sequence, manufacturing time, an intra-cellular layout of unequal-area facilities, and part scheduling with the aim of minimization of makespan, tardiness penalties, and transportation costs. Wang et al. [63] presented a novel linear mathematical model integrating cell formation and task scheduling with the dual-resource constrained setting in a CMS. They proposed a hybrid SA to minimize the makespan of manufacturing project in a CMS.

\subsection{CMS studies with a SA solution approach}

To solve the cell formation problem, Pailla et al. [47] presented an evolutionary algorithm and a SA. A known set of CFP instances were used to evaluate the performance of both algorithms. Also, the results of both algorithms were compared with those of five other algorithms. Generally, SA outperformed the proposed evolutionary algorithm and the other five algorithms. Kia et al. [26] formulated an MINLP model for the machines layout design in a DCMS. A new feature of their model was simultaneously making the CF and Group Layout (GL) decisions though a dynamic environment and considering the multi-rows layout for machines location in the flexibly-configured cells.

Bayram et al. [8] addressed the DCMS design and formulated a mathematical model. The objective function was to minimize inter-cell and intra-cell material handling, machine purchasing, layout reconfiguration, variable and constant machine costs. They designed two heuristic solution approaches that combine SA with Linear Programming (LP) and GA with LP. Liu and Wang [33] designed a non-linear integer mathematical model for a CMS with multi-functional machines and the multi-skilled workers need to be grouped and assigned to the cells. To minimize the makespan, a hybrid SA embedding priority rule-based heuristic algorithm was proposed. To improve grouping efficacy of a cell formation problem, Zeb et al. [64] presented the hybridization of SA with GA to combine exploration power of GA with the intensification power of SA. Ghosh et al. [21] proposed a Quadratic Assignment Problem (QAP) model of the inter-cell layout design problem in a dynamic environment considering the material handling cost and a modified cells proximity relationship. Due to the NP-hard nature of the problem, they developed an improved GA and a SA algorithm. Results showed that both techniques are viable in terms of solution quality and computational time. Defersha and Hodiya [12] developed a parallel multiple search path SA to solve a mathematical model integrating distributed layout design and cell formation with the aim of minimization of a weighted sum of material handling and inter-cell movement costs. Shafigh et al. [56] presented linear programming embedded SA for solving a comprehensive model in the design and operation of the distributed layout-based manufacturing systems. Their mathematical model incorporates important manufacturing attributes including demand fluctuation, system reconfiguration, lot splitting, workload balancing, alternative routing, machine capability, tooling requirements, material handling cost, machine relocation cost, setup cost, inventory carrying cost, in-house production, and subcontracting costs.

Iqbal and Al-Ghamdi [24] developed a nonlinear mathematical model to optimize the assignment of manufacturing processes to various machines and grouping machines in cells for minimizing parts transportation distance. A SA algorithm was developed to save production and transportation energies by assigning the parts operations to the most appropriate machines and optimally grouping the machines into cells. Feng et al. [17] developed an MILP model to integrate cell formation and layout problems. The model featured the simultaneous incorporation of some CMSs design attributes including unequal machine dimensions, duplicate machines, alternative process routings, lot splitting, and PP. They proposed two hybrid approaches, one combining GA and LP and the other combining SA and LP. Rafiei et al. [51] proposed an MINLP model to address the cell formation problem and job scheduling simultaneously to minimize the costs of operations and transportation. They developed a hybrid SA/GA.

Out of 39 papers reviewed in this section, SA is a prevalent solution approach employed by many researchers due to its strength in handling complex problems, especially with numerous constraints which are common in CMS models.

It is evident from the literature that every structural and operational decision parameter contributes somehow for improving the production efficiency and the integration of all them in the CMS design would enhance the application potential in the discrete part manufacturing sector (e.g., automobile and machine tool manufacturers). On these considerations, this paper formulates a DCMS model to take decisions on the following 
structural and operational decision parameters in an integrated manner: (1) parts transportation planning, (2) raw materials orders planning, (3) machines assignments to cells in a reconfigurable environment, (4) parts operations assignment to machines, (5) parts operations scheduling, and (6) PP strategies planned for each part type.

The integrated model presents a larger coverage of the manufacturing characteristics. Also, a larger collection of input parameters, decision variables and objective function components related to CM is incorporated than the reviewed studies.

\section{MAThEMATICAL MODEL AND PROBLEM DESCRIPTIONS}

\subsection{Model assumptions}

In this section, the DCMS model integrating CF, MRP, parts scheduling, GFCTP and PP is formulated as an MINLP model under the following assumptions:

(1) It is assumed that there is a deterministic volume for the demand of each part type to be satisfied at each market in each period. Also, regarding the fluctuations of the production levels based on the given demand volumes and manufacturing-related costs, the system can manufacture some extra parts in a given period, keep them as inventories in the markets warehouse between consecutive periods and used them in the upcoming periods with high demand level. Also, because of the limited machine capacities, backordering can be employed to postpone the fulfillment of the markets demands after their known due dates.

(2) There are different vehicles for transporting parts from the plant to the markets with different costs and speeds.

(3) For each part, due date at each market in each period is known. As a result, a tardiness penalty is imposed for a part if it is not delivered to the market on its due date in the intended demand period.

(4) Parts can be produced one or some periods earlier than their due date. After production, the produced parts should be carried to the markets warehouse immediately since holding finished parts in the plant warehouse is not allowed during a period.

(5) To transport the products required by a specific market, it is not necessary to complete the manufacturing of all products required by that market for simultaneous transportation. So, parts might be transported to a market by separate delivery. In other words, each product should be sent to the demanding market immediately after finishing the process of its last operations.

(6) The usage coefficient of each raw material in a part is given with a one-level BOM. Also, the lead time for each raw material is different.

(7) There are some raw materials in the plant warehouse in the first period.

(8) The order cost for each raw material in each period is considered.

(9) Raw materials can be ordered and received earlier than the required period. As a result, they should be kept in the plant warehouse incurring inventory holding cost.

(10) It is assumed that there is only one duplicate of each machine type with a fixed capacity.

(11) Upper and lower size of each cell in terms of assigned machines is known.

(12) Parts are transported individually by the material handling equipment inside and among the cells referred to as intracellular and intercellular movements, respectively. The time for each intracellular and intercellular movement of each part is different; however, the cost is considered the same for all parts.

(13) There are several operations for each part type to be processed respect to its sequence data.

(14) It is considered that all machine types are multi-purposed and capable of manufacturing one or more operations with no reinstallation cost. In other meaning, each part operation can be manufactured by different machines with different manufacturing times. Although, each operation should be manufactured by only one of those machines which are capable of manufacturing that operation and splitting the production lot for a part operation is not permitted. 
(15) Machines are not allowed to manufacture more than one part operation simultaneously.

(16) Cell reconfiguration involves the situations where the existing machines between different cells are relocated due to fluctuating capacity requirements during the successive periods. The cost of machine relocation is calculated based on the number of machines relocated among cells. Relocating a machine includes removing $f$ a machine from a cell and adding that to another cell. The relocation cost is different for each machine type.

(17) After starting the process of an operation by a machine, no interruption is permitted.

(18) To process some part operations on a machine, it is considered the sequence, in which part operations are assigned to a machine. For scheduling problem in this model, some time positions for each machine are considered, i.e., for each part operation manufactured by the machine a time position is occupied.

In fact, the main purpose of the developed model is to assess the effect of simultaneous integration of $\mathrm{CF}$, MRP, scheduling, GFCTP and PP in designing a DCMS into a transportation network regarding to the burdened total costs of holding the parts inventories in the markets, backorders, tardiness, transportation of the parts from the plant to the markets, purchase of raw materials, keeping raw materials in the plant warehouse, intercellular and intracellular movements and machine relocation.

The main constraints of the formulated model are imposed by the limitations of (1) machines process capability, (2) sequencing time positions, (3) manufacturing each operation, (4) maximum and minimum cell size, (5) machine relocation, (6) machine time-capacity, (7) relation between starting times and finishing times of the operations, (8) tardiness calculations, (9) markets demand satisfaction, (10) raw materials requirements planning and (11) transportation vehicles capacity.

$$
\begin{array}{ll}
\text { Sets } & \\
\quad j=\{1,2, \ldots, J\} & \text { index for markets. } \\
i=\{1,2, \ldots, I\} & \text { index for vehicles. } \\
r=\{1,2, \ldots, R\} & \text { index for raw materials. } \\
h=\{1,2, \ldots, H\} & \text { index for periods. } \\
p=\{1,2, \ldots, P\} & \text { index for part types. } \\
k=\{1,2, \ldots, K\} & \text { index for operations. } \\
c=\{1,2, \ldots, C\} & \text { index for cells. } \\
m=\{1,2, \ldots, M\} & \text { index for machine types. } \\
b=\{1,2, \ldots, B\} & \text { index for time positions. }
\end{array}
$$

\author{
Model parameters \\ $d u_{p j h} \quad$ due date of part $p$ demanded by market $j$ during period $h$. \\ $\pi_{p j h} \quad$ inventory holding cost of part $p$ in the warehouse of market $j$ in the case of being received before \\ demand period $h$. \\ $g_{p j h}^{\prime} \quad$ backorder cost of part type $p$ demanded by market $j$ in period $h$. \\ $\beta_{p j h} \quad$ the unitary tardiness penalty cost of part $p$ demanded by market $j$ during period $h$. \\ $D_{p j h} \quad$ demand of market $j$ for part type $p$ in period $h$. \\ $D_{p j h}^{\prime} \quad$ if demand of market $j$ for part type $p$ in period $h$ is positive (i.e., $D_{p j h}>0$ ); 0 otherwise. \\ $f_{i j} \quad$ required time for vehicle type $i$ for transporting parts from the plant to market $j$. \\ $\varphi_{i j h} \quad$ cost of transporting parts from the plant to market $j$ by vehicle $i$ in period $h$. \\ $\omega_{p r} \quad$ the usage coefficient of raw material $r$ in part type $p$. \\ $\mathrm{LT}_{r} \quad$ lead time for raw material $r$. \\ $\sigma_{r h} \quad$ order cost per unit of raw material $r$ in period $h$. \\ $\mu_{r h} \quad$ holding cost of raw material $r$ during period $h$ in the plant warehouse. \\ IS $_{p r} \quad$ initial level of raw material $r$ available to be used in part type $p$. \\ $u_{m h} \quad$ relocation cost of machine type $m$ in period $h$. \\ $B_{u} \quad$ upper limit for cell size.
}


$B_{l} \quad$ lower limit for cell size.

$\mathrm{TM}_{m h} \quad$ capacity of machine type $m$ during period $h$.

$\mathrm{IE}_{p} \quad$ intercellular movement time for part type $p$.

$\mathrm{IA}_{p} \quad$ intracellular movement time for part type $p$.

$\gamma_{h} \quad$ cost of inter-cellular movement per time unit in period $h$.

$\lambda_{h} \quad$ cost of intra-cellular movement per time unit in period $h$.

$e_{k p m} \quad$ manufacturing time of one unit of operation $k$ of part $p$ by machine $m$.

$q_{k p m} \quad$ if operation $k$ of part $p$ is allowed to be manufactured by machine $m$; 0 otherwise.

$M \quad$ an arbitrary big positive number.

Decision variables

$T_{p j h} \quad$ the amount of tardiness happening in delivering part type $p$ demanded by market $j$ during period $h$.

$y_{i p j h h^{\prime}} \quad$ if part type $p$ demanded by market $j$ in period $h$ is transported by vehicle $i$ in period $h^{\prime}$; 0 otherwise.

$O_{r p h} \quad$ the amount of order for raw material $r$ received in period $h$ to be used in part type $p$.

$W_{m c h}$

$K_{m c h}^{+}$ if machine type $m$ is assigned to cell $c$ in period $h ; 0$ otherwise.

$K_{m}^{-}$ch if machine type $m$ is added to cell $c$ in period $h$.

$Z_{\text {kpjmbhh' }}$ if machine type $m$ is removed from cell $c$ in period $h$.

$S_{k p j m b h h^{\prime}} \quad$ the start time of manufacturing operation $k$ of part type $p$ manufactured by machine $m$ at if operation $k$ of part type $p$ demanded by market $j$ in period $h$ is manufactured by machine $m$ at its time position $b$ in period $h^{\prime} ; 0$ otherwise. its time position $b$ in period $h^{\prime}$ and demanded by market $j$ in period $h$.

$c_{\text {com }}$ thmbh $^{\prime}$ the ending time of manufacturing operation $k$ of part type $p$ manufactured by machine $m$ at its time position $b$ in period $h^{\prime}$ and demanded by market $j$ in period $h$.

$C_{p j h h^{\prime}} \quad$ the completion time of manufacturing all operations of part type $p$ produced in period $h^{\prime}$ and demanded by market $j$ in period $h$.

\subsection{Mathematical model}

$$
\begin{aligned}
\operatorname{Min}: & \sum_{p=1}^{P} \sum_{j=1}^{J} \sum_{m=1}^{M} \sum_{b=1}^{B} \sum_{h=1}^{H} \sum_{h^{\prime}=1}^{H} \sum_{h^{\prime} \leq h^{\prime \prime}<h} Z_{K p j m b h h^{\prime}} \cdot \pi_{p j h^{\prime \prime}} \\
& +\sum_{p=1}^{P} \sum_{j=1}^{J} \sum_{m=1}^{M} \sum_{b=1}^{B} \sum_{h=1}^{H} \sum_{h^{\prime}=1}^{H} \sum_{h \leq h^{\prime \prime}<h^{\prime}} Z_{K p j m b h h^{\prime}} g_{p j h^{\prime \prime}}^{\prime} \\
& +\sum_{p=1}^{P} \sum_{j=1}^{J} \sum_{h=1}^{H} \beta_{p j h} T_{p j h} \\
& +\sum_{i=1}^{I} \sum_{p=1}^{P} \sum_{j=1}^{J} \sum_{h=1}^{H} \sum_{h^{\prime}=1}^{H} y_{i p j h h^{\prime}} \cdot \varphi_{i j h^{\prime}} \\
& +\sum_{r=1}^{R} \sum_{p=1}^{P} \sum_{h=1}^{H} O_{r p h} \sigma_{r h} \\
& +\sum_{p=1}^{P} \sum_{r=1}^{R}\left[\sum_{h^{\prime}=1}^{H} \mu_{r h^{\prime}}\left(\mathrm{IS}_{p r}-\sum_{h^{\prime \prime} \leq h^{\prime}} \sum_{j=1}^{J} \sum_{h=1}^{H} \sum_{m=1}^{M} \sum_{b=1}^{B} Z_{K p j m b h h^{\prime \prime}} D_{p j h} \cdot \omega_{p r}\right)\right. \\
& \left.+\sum_{h^{\prime} \geq \mathrm{LT}_{r}+1}^{H} \sum_{h^{\prime \prime} \leq h^{\prime}-\mathrm{LT}_{r}}^{H} \mu_{r h^{\prime}} O_{r p h^{\prime \prime}}\right]
\end{aligned}
$$




$$
\begin{aligned}
& +\sum_{k=1}^{K} \sum_{p=1}^{P} \sum_{j=1}^{J} \sum_{m=1}^{M} \sum_{m^{\prime}=1}^{M} \sum_{b=1}^{B} \sum_{b^{\prime}=1}^{B} \sum_{c=1}^{C} \sum_{\substack{c^{\prime}=1 \\
c^{\prime} \neq c}}^{C} \sum_{h=1}^{H} \sum_{h^{\prime}=1}^{H} \gamma_{h^{\prime}} \\
& \times \mathrm{IE}_{p} \cdot Z_{k p j m b h h^{\prime}} \cdot W_{m c h^{\prime}} \cdot Z_{(k+1) p j m^{\prime} b^{\prime} h h^{\prime}} \cdot W_{m^{\prime} c^{\prime} h^{\prime}} \cdot D_{p j h} \\
& +\sum_{k=1}^{K} \sum_{p=1}^{P} \sum_{j=1}^{J} \sum_{m=1}^{M} \sum_{\substack{m^{\prime}=1 \\
m^{\prime} \neq m}}^{M} \sum_{b=1}^{B} \sum_{b^{\prime}=1}^{B} \sum_{c=1}^{C} \sum_{h=1}^{H} \sum_{h^{\prime}=1}^{H} \lambda_{h^{\prime}} \\
& \times \mathrm{IA}_{p} \cdot Z_{k p j m b h h^{\prime}} \cdot W_{m c h^{\prime}} \cdot Z_{(k+1) p j m^{\prime} b^{\prime} h h^{\prime}} \cdot W_{m^{\prime} c h^{\prime}} \cdot D_{p j h} \\
& +\sum_{m=1}^{M} \sum_{c=1}^{C} \sum_{h^{\prime}=1}^{H}\left(K_{m c h^{\prime}}^{+}+K_{m c h^{\prime}}^{-}\right) u_{m h^{\prime}}
\end{aligned}
$$

s.t.

$$
\begin{aligned}
& \sum_{m=1}^{M} \sum_{b=1}^{B} \sum_{h^{\prime}=1}^{H} q_{k p m} Z_{k p j m b h h^{\prime}}=D_{p j h}^{\prime} \quad \forall k, p, j, h \\
& \sum_{m=1}^{M} \sum_{b=1}^{B} Z_{(k+1) p j m b h h^{\prime}} \leq \sum_{m=1}^{M} \sum_{b=1}^{B} Z_{k p j m b h h^{\prime}} \quad \forall k<K, p, j, h, h^{\prime} \\
& \sum_{k=1}^{K} \sum_{p=1}^{P} \sum_{j=1}^{J} \sum_{h=1}^{H} Z_{k p j m b h h^{\prime}} \leq 1 \quad \forall m, b, h^{\prime} \\
& \sum_{m=1}^{M} W_{m c h^{\prime}} \leq B_{u} \quad \forall c, h^{\prime} \\
& \sum_{m=1}^{M} W_{m c h^{\prime}} \geq B_{l} \quad \forall c, h^{\prime} \\
& \sum_{c=1}^{C} W_{m c h^{\prime}}=1 \quad \forall m, h^{\prime} \\
& W_{m c\left(h^{\prime}-1\right)}+K_{m c h^{\prime}}^{+}-K_{m c h^{\prime}}^{-}=W_{m c h^{\prime}} \quad \forall m, c, h^{\prime}>1 \\
& \sum_{k=1}^{K} \sum_{p=1}^{P} \sum_{j=1}^{J} \sum_{b=1}^{B} \sum_{h=1}^{H} Z_{k p j m b h h^{\prime}} \cdot D_{p j h} \cdot e_{k p m} \leq \sum_{c=1}^{C} \mathrm{TM}_{m h^{\prime}} \cdot W_{m c h^{\prime}} \quad \forall m, h^{\prime} \\
& S_{k p j m b h h^{\prime}} \geq\left(\begin{array}{l}
\operatorname{com}_{(k-1) p j m^{\prime} b^{\prime} h h^{\prime}}+\mathrm{IE}_{p} \cdot\left(W_{m c h^{\prime}}+W_{m^{\prime} c^{\prime} h^{\prime}}-1\right) \\
-M \cdot\left(1-Z_{k p j m b h h^{\prime}}\right)
\end{array}\right) \quad \forall k>1, p, j, m \neq m^{\prime}, b, b^{\prime}, c \neq c^{\prime}, h, h^{\prime} \\
& S_{k p j m b h h^{\prime}} \geq\left(\begin{array}{l}
\operatorname{com}_{(k-1) p j m^{\prime} b^{\prime} h h^{\prime}}+\mathrm{IA}_{p} \cdot\left(W_{m c h^{\prime}}+W_{m^{\prime} c h^{\prime}}-1\right) \\
-M \cdot\left(1-Z_{k p j m b h h^{\prime}}\right)
\end{array}\right) \quad \forall k>1, p, j, m \neq m^{\prime}, b, b^{\prime}, c, h, h^{\prime} \\
& S_{k p j m b h h^{\prime}} \geq \operatorname{com}_{(k-1) p j m b^{\prime} h h^{\prime}}-M \cdot\left(1-Z_{k p j m b h h^{\prime}}\right) \quad \forall k>1, p, j, m, b, b^{\prime}, h, h^{\prime} \\
& S_{k p j m b h h^{\prime}} \geq \operatorname{com}_{k^{\prime} p^{\prime} j^{\prime} m(b-1) h h^{\prime}}-M \cdot\left(1-Z_{k p j m b h h^{\prime}}\right) \quad \forall k, k^{\prime}, p \neq p^{\prime}, j, j^{\prime}, m, b>1, h, h^{\prime} \\
& S_{k p j m b h h^{\prime}} \geq \operatorname{com}_{k^{\prime} p^{\prime} j^{\prime} m(b-1) h^{\prime \prime} h^{\prime}}-M \cdot\left(1-Z_{k p j m b h h^{\prime}}\right) \quad \forall k, k^{\prime}, p, p^{\prime}, j, j^{\prime}, m, b>1, h \neq h^{\prime}, h^{\prime} \\
& \operatorname{com}_{k p j m b h h^{\prime}}=S_{k p j m b h h^{\prime}}+e_{k p m} \cdot Z_{k p j m b h h^{\prime}} \cdot D_{p j h} \quad \forall k, p, j, m, b, h, h^{\prime} \\
& S_{k p j m b h h^{\prime}} \leq M \cdot Z_{k p j m b h h^{\prime}} \\
& \forall k, p, j, m, b, h, h^{\prime} \\
& \text { com }_{k p j m b h h^{\prime}} \leq M \cdot Z_{k p j m b h h^{\prime}}
\end{aligned}
$$




$$
\begin{aligned}
& C_{p j h h^{\prime}}=\sum_{m=1}^{M} \sum_{b=1}^{B} \operatorname{com}_{K p j m b h h^{\prime}} \cdot D_{p j h}^{\prime} \quad \forall p, j, h, h^{\prime} \\
& \left(C_{p j h h}+\sum_{i=1}^{I} y_{i p j h h} f_{i j}\right)-d u_{p j h} \leq T_{p j h} \quad \forall p, j, h \\
& \sum_{i=1}^{I} \sum_{h^{\prime}=1}^{H} y_{i p j h h^{\prime}}=D_{p j h}^{\prime} \quad \forall p, j, h \\
& D_{p j h}=\sum_{m=1}^{M} \sum_{b=1}^{B} \sum_{h^{\prime}=1}^{H} Z_{k p j m b h h^{\prime}} \cdot D_{p j h} \quad \forall k, p, j, h \\
& \sum_{j=1}^{J} \sum_{m=1}^{M} \sum_{b=1}^{B} \sum_{h=1}^{H} Z_{K p j m b h h^{\prime}} D_{p j h} \cdot \omega_{p r} \leq \mathrm{IS}_{p r} \quad \forall p, r, h^{\prime}=1 \\
& \sum_{j=1}^{J} \sum_{m=1}^{M} \sum_{b=1}^{B} \sum_{h=1}^{H} Z_{K p j m b h h^{\prime}} D_{p j h} \cdot \omega_{p r} \\
& \leq \mathrm{IS}_{p r}-\sum_{j=1}^{J} \sum_{m=1}^{M} \sum_{b=1}^{B} \sum_{h=1}^{H} \sum_{h^{\prime \prime}<h^{\prime}} Z_{K p j m b h h^{\prime \prime}} D_{p j h} \cdot \omega_{p r} \quad \forall p, r, 2 \leq h^{\prime} \leq \mathrm{LT}_{r} \\
& \sum_{j=1}^{J} \sum_{m=1}^{M} \sum_{b=1}^{B} \sum_{h=1}^{H} Z_{K p j m b h h^{\prime}} D_{p j h} \cdot \omega_{p r} \\
& \leq \mathrm{IS}_{p r}+\sum_{h^{\prime \prime} \leq h^{\prime}-\mathrm{LT}_{r}} O_{r p h^{\prime \prime}} \\
& -\sum_{j=1}^{J} \sum_{m=1}^{\bar{M}} \sum_{b=1}^{B} \sum_{h=1}^{H} \sum_{h^{\prime \prime}<h^{\prime}} Z_{K p j m b h h^{\prime \prime}} D_{p j h} \cdot \omega_{p r} \\
& \sum_{m=1}^{M} \sum_{b=1}^{B} D_{p j h}^{\prime} \cdot Z_{K p j m b h h^{\prime}}=\sum_{i=1}^{I} y_{i p j h h^{\prime}} \quad \forall p, j, h, h^{\prime}
\end{aligned}
$$

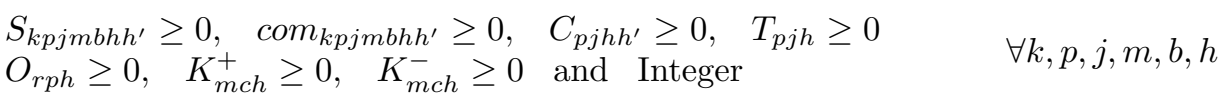

$$
\begin{aligned}
& Z_{k p j m b h h^{\prime}}, \quad W_{m c h}, \quad y_{i p j h h^{\prime}} \in\{0,1\} \quad \forall i, k, p, j, m, b, h .
\end{aligned}
$$

The objective function consists of nine terms. Term (3.1a) deals with the computation of inventory holding cost for parts in the case of being produced in a period $\left(h^{\prime}\right)$ earlier than the demand period $h\left(\right.$ i.e., $\left.h^{\prime}<h\right)$. Term (3.1b) computes the backorder cost for parts in the case of being produced in a period $\left(h^{\prime}\right)$ later than the demand period $h\left(\right.$ i.e., $\left.h^{\prime}>h\right)$. Term (3.1c) calculates tardiness cost imposed for a part if it is received by the market after its due date in the intended demand period. Term (3.1d) calculates the transportation cost for the parts to be transported from the plant to the markets by heterogeneous vehicles. Term (3.1e) computes the raw materials purchase cost. Term (3.1f) calculates holding cost of raw materials in the plant warehouse Terms $(3.1 \mathrm{~g})$ and $(3.1 \mathrm{~h})$ address intercellular and intracellular movement costs computation, respectively. Term (3.1i) calculates the machines relocation costs.

Constraint (3.2) guarantees that in the case of non-zero demand by a market for a part type, each operation of that part is manufactured only at one time position of a machine capable of manufacturing that part operation. Constraint (3.3) ensures that all operations of a part are manufactured based on the sequence of the operations. Constraint (3.4) ensures that at most one part operation can be processed at each time position of a machine. Constraints (3.5) and (3.6) support that the number of machines assigned to a cell does not exceed the lower and 
upper limit. Constraint (3.7) states that each machine is assigned to only one cell during a period. Constraint (3.8) is the machine balance constraint checking if a machine is relocated between two cells during two successive periods. Constraint (3.9) obliges to not exceeding the machines capacity limits.

Constraint (3.10) assumes that in the case of intercellular movement, the starting time of processing a part operation has to be higher than of the sum of the completion time of the predecessor operation plus the corresponding intercellular movement time. Similarly, Constraint (3.11) assumes that in the case of intracellular movement, the starting time of a part operation has to be higher than of the sum of the completion time of the predecessor operation plus the corresponding intracellular movement time. Constraint (3.12) supports that in case of two consecutive operations of a part being manufactured by the same machine, the starting time of the successor operation is higher than the completion time of the predecessor operation.

Constraints (3.13) and (3.14) guarantee that the starting time of manufacturing a part operation processed at time position $b$ by machine $m$ should be larger than the completion time of another part operation processed at earlier time position $(b-1)$ of that machine. Constraint (3.15) indicates that the completion time of a part operation equals the sum of its starting time plus its manufacturing time. According to constraints (3.16) and (3.17), decision variables $S_{k p j m b h h^{\prime}}$ and $\operatorname{com}_{k p j m b h h^{\prime}}$ calculating starting and ending time of operation $k$ of part $p$ performed at time position $b$ of machine $m$ could take a positive value if processing that operation is performed at corresponding time position of that machine. Constraint (3.18) computes the completion time of a part which is equal to the ending time of manufacturing the last operation of that part. Constraint (3.19) calculates the tardiness amount for a part which is equal to the deviation between its due date and its delivery time to the relevant market (i.e., the sum of its completion time and its transportation time from the plant to the relevant market). Constraint (3.20) reiterates if there is a demand for a part by a market, it should be transported by one of the vehicles. Equation (3.21) is the demand satisfaction constraint. The demand for a market in a period has to be satisfied using the internal production in one of the time periods that may be one or more periods earlier than the intended demand period as inventory holding strategy, one or more periods later than the intended demand period as backorders strategy and or in the same period without incurring holding or backordering cost. A novelty in the present model is employing the PP strategies (i.e., inventory holding and backorder) without introducing extra decision variables for calculating the number of inventories and backorders. This is achieved by considering parameters $h$ and $h^{\prime}$ representing demand period and production period in decision variable $Z_{\text {kpjmbhh' }}$, respectively.

Constraint (3.22) states that the amount of used raw materials calculated based on the production level in the first period should be less than the initial inventory level of required raw materials. Constraint (3.23) enforces that the amount of raw material $r$ used in the production of part type $p$ between the second period and period $h^{\prime}$ which is earlier than the period $\mathrm{LT}_{r}$ (i.e., its lead time) should be less than the inventory level of raw material $r$ available in the plant warehouse at period $h^{\prime}$ (i.e., the initial inventory level $\mathrm{IS}_{p r}$ minus the amount of raw material $r$ used for the production of part type $p$ during periods 2 to $h^{\prime}$ ). Similarly, Constraint (3.24) asserts that the amount of raw material $r$ used for the production of part type $p$ in period $h^{\prime}$ after its lead time should be lower than the sum of its initial inventory and the amount of received orders minus the amount of raw material used in the prior periods. It is worth mentioning the raw material $r$ ordered in period $h$ is received by the plant in period $\mathrm{LT}_{r}+h$. Constraint (3.25) indicates that the parts produced in period $h^{\prime}$ to satisfy demand at period $h$ have to be delivered to one of the markets in the same production period $h^{\prime}$ since holding of finished parts is not permitted by the plant warehouse. Constraints (3.26) and (3.27) necessitate the logical binary and non-negativity integer conditions for the model decision variables.

Since Terms $(3.1 \mathrm{~g})$ and $(3.1 \mathrm{~h})$ in the objective function are nonlinear, the auxiliary decision variable $X_{k p j m m^{\prime} b b^{\prime} c c^{\prime} h h^{\prime}}$ is defined and replaced with a term $Z_{k p j m b h h^{\prime}} \cdot W_{m c h^{\prime}} \cdot Z_{(k+1) p j m^{\prime} b^{\prime} h h^{\prime}} \cdot W_{m^{\prime} c^{\prime} h^{\prime}}$ in Terms $(3.1 \mathrm{~g})$ and $(3.1 \mathrm{~h})$ and the following constraint is also added to the main model.

$$
X_{k p j m m^{\prime} b b^{\prime} c c^{\prime} h h^{\prime}} \geq W_{m c h^{\prime}}+Z_{k p j m b h h^{\prime}}+W_{m^{\prime} c^{\prime} h^{\prime}}+Z_{(k+1) p j m^{\prime} b^{\prime} h h^{\prime}}-3.5 \begin{aligned}
& \forall k<K, p, j, m \neq m^{\prime}, \\
& b, b^{\prime}, c, c^{\prime}, h, h^{\prime}
\end{aligned} .
$$




$$
\left.\left\langle(\text { Ma_Ce })_{m \times c}\right|(\text { Order })_{r \times p} \mid(\text { Production })_{(k+1) \times 5 \times x}\right\rangle
$$

FIGURE 1. Solution structure representation.

$$
M a \_C e=\left(\begin{array}{cccc}
x_{11} & x_{12} & \ldots & x_{1 c} \\
x_{21} & x_{22} & \ldots & x_{2 c} \\
\vdots & & & \\
x_{m 1} & x_{m 2} & \ldots & x_{m c}
\end{array}\right)
$$

Figure 2. Assignment of machines to cells.

\section{Simulated annealing Algorithm}

For solving hard combinatorial optimization problems, the Simulated Annealing (SA) algorithm was designed by Kirkpatrick et al. [30] as a stochastic neighborhood search method to simulate the annealing process directing a system to its lowest energy level by the assistance of a controlled cooling scheme. Regularly, the annealing process takes place as follows: (1) the temperature is raised to an adequate level, (2) it is kept in each level for a suitable period, and (3) it is reduced under the controlled conditions until reaching the determined energy level.

The goal behind the SA approach is to avoid local optimums using temperature changes and non-improving solutions acceptance strategy (up/downhill search) in a discrete environment. SA approach has several merits compared with other meta-heuristic ones as the following: (1) not being highly dependent on the initial point, (2) having the potential to escape local optimums, (3) the satisfactory performance in discrete optimization, (4) a suitable instrument to address the problems with continuous solution space, (5) simple in terms of programming and execution, and (6) it has been proved that its computational time has an upper limit of a certain polynomial type in terms of problem dimensions [30].

SA has been applied in numerous optimization problems in a considerable diversity of areas, including DCMS $[8,12,17,21,24,26,28,29,33,37,39,42,51,56,64]$. In this section, the elements of the extended SA are described as follows.

\subsection{Solution structure representation}

Solution structure indicates a point of feasible solution space so that its representation manner in each metaheuristic approach is important. Solution structure in the proposed SA algorithm for the presented model as depicted in Figure 1 has been made up of three parts as follows.

In this representation, $m$ is the machine type index set, $c$ is the index of the cell, $r$ is the raw materials index, $p$ is the parts types index and $k$ is for operation index. Also, index $x$ belongs to the third dimension of Production matrix. In the continuation, each of these matrices has been explained.

The first part as matrix $M a_{-} C e$ with $m \times c$ binary elements states each machine assignment to a unique cell and its detailed representation is depicted in Figure 2.

In this matrix, each row shows the type of the utilized machine and each column stands for the considered cell. Since there is only one duplicate of each machine type, thus in each row, there is only one non-zero element. Also, it has to be noticed that the number of machines assigned to each cell should be placed in the cell size limit. As a result, the number of ones in each column follows the following rule:

$$
B_{1} \leq \text { the number of machines assigned to a cell (the number of } 1 \mathrm{~s} \text { in each column) } \leq B_{u} \text {. }
$$




$$
M a \_C e=\left(\begin{array}{ll}
0 & 1 \\
1 & 0 \\
0 & 1
\end{array}\right)
$$

Figure 3. Example for Matrix $M a_{-} C e$.

$$
\begin{gathered}
\text { Order }=\left(\begin{array}{cccc}
x_{11} & x_{12} & \ldots & x_{1 p} \\
x_{21} & x_{22} & \ldots & x_{2 p} \\
\vdots & & & \\
x_{r 1} & x_{r 2} & \ldots & x_{p p}
\end{array}\right) \\
\text { Order }=\left(\begin{array}{ccc}
25 & 0 & 10 \\
12 & 0 & 0 \\
0 & 18 & 20
\end{array}\right)
\end{gathered}
$$

Figure 4. Matrix Order (order amount of each raw material) with an example.

$$
\text { Production }=\left(\begin{array}{ccccc}
p & h & j & \text { dem } & i \\
m_{i_{1}} & T_{\text {transport }_{1}} & T_{\text {begin }_{1}} & T_{\text {process }_{1}} & T_{\text {end }_{1}} \\
\vdots & & \ddots & & \vdots \\
m_{i_{k}} & T_{\text {transport }_{k}} & T_{\text {begin }_{k}} & T_{\text {process }_{k}} & T_{\text {end }_{k}}
\end{array}\right)
$$

Figure 5. Matrix Production.

The matrix $M a_{-} C e$ satisfies constraints (3.6) to (3.8) in the main model in each period. Also, the set of matrices $M a_{-} C e$ available in all periods has to meet constraint (3.9). As an example for Matrix $M a_{-} C e$ depicted in Figure 3, the machine type 2 is assigned to cell 1 and machines of type 1 and 3 to cell 2.

The second part as matrix Order with $r \times p$ integer elements displays the order amount of each raw material for each product as depicted in Figure 4.

The receiving time of an ordered raw material depends on its lead time. To minimize inventory holding cost, the maximum ordered raw material amount in all periods is determined based on their related products so that in the last period, the minimum raw material amount remains in the warehouse. This matrix satisfies constraints (3.23) to (3.25) in each period. As an example for Matrix Order depicted in Figure 4, 18 units of raw material type 2 and 20 units of raw material type 3 for the 3rd part have been ordered.

The third part as matrix Production with $(k+1) \times 5 \times x$ elements as depicted in Figure 5, viewed as the major part of the chromosome, contains all manufacturing data of each feasible solution. The 3rd dimension of this matrix does not have any certain size and would take different values proportionate with the existing capacity in each period.

The 1st row of matrix Production includes five indices as part type $(p)$, period $(h)$, market $(j)$, requested demand amount $(\mathrm{dem})$ and vehicle number $(i)$. The subsequent rows belong to operations 1 to $k$, whose elements are respectively the manufacturing machine number $(m)$, intra- or inter-cell movement time $\left(T_{\text {transport }}\right)$, the starting 


$$
\begin{aligned}
& \text { Production(:,:,1) }=\left(\begin{array}{ccccc}
2 & 1 & 1 & 15 & 1 \\
3 & 0 & 0 & 90 & 90 \\
3 & 0 & 90 & 60 & 150 \\
2 & 7 & 157 & 20 & 177
\end{array}\right) \\
& \text { Production(:,:,2)}=\left(\begin{array}{ccccc}
1 & 3 & 2 & 20 & 2 \\
2 & 0 & 0 & 40 & 40 \\
1 & 9 & 49 & 80 & 129 \\
3 & 3 & 150 & 60 & 210
\end{array}\right)
\end{aligned}
$$

Figure 6. Example of matrix Production for two parts.

time of manufacturing operation $\left(T_{\text {begin }}\right)$, total manufacturing time $\left(T_{\text {process }}\right)$ and the completion time of manufacturing operation $\left(T_{\text {end }}\right)$. All the remaining constraints in the main model are met by this matrix. An example of matrix Production for two parts is depicted in Figure 6 and their manufacturing procedure is described as follows.

First, part 2 is loaded on the production line in period 1 as it is demanded by market 1 at the demand level equal to 15 . The 1 st operation is done by machine 3 and since it is the 1 st operation, its handling cost is 0 . The operation has started at time 0 on machine 3 and since its manufacturing time is 90 , its process finishes at time 90 . The 2 nd operation is done on the same machine. Thus, it incurs no handling cost. Then, the 2 nd operation immediately starts on machine 3 and regarding its 60 unit processing time, its process finishes at time 150. Operation 3 must be performed on machine 2 and as a result, intercellular or intracellular movement is required. This movement type is defined based on matrix $\mathrm{Ma} a_{-} \mathrm{Ce}$ and its related number is derived from the input values in the previous example. Because the 2 nd operation has finished at time 150 and its movement time equals 7 , the $3 \mathrm{rd}$ operation started at time 157 and considering its processing time 20 units, it terminates at time 177. The produced part is immediately transported to market 1 by vehicle type 1 that its time and transportation cost are derived from the input values and accounted in the objective function.

The next part manufactured in this period is part 1 requested by market 2 with 20 units demand for the 3rd period. The 1st operation is done on machine 2 during 40-time units. For processing the 2nd operation on machine 1, its movement time is considered as 9 units from the input values based on matrix $M a_{-} C e$ and therefore, the operation starts at time 49 and finishes at time 129 regarding the 80-time units required for its processing. The 3rd operation must be performed on machine 3. Regarding movement time equal to 3 units, the operation can begin at time 132. However, this machine is busy with another part until time 150. Therefore, the current part has to wait in a queue until machine 3 is unloaded. Afterwards, part 1 is loaded on machine 3 and since its processing time is 60 units, the processing of the last operation finishes at time 210. Finally, part 1 is transported to market 2 by vehicle 2 . It should be noticed that the aforementioned matrices belong to one period. Thus, in each solution, there is $T$ set of these matrices where $T$ stands for the number of periods.

\subsection{Initial solution generation}

An initial solution as a starting point employed in the search process is generated according to a hierarchical approach in four stages In this approach, matrices $\left[\mathrm{Ma} a_{-} \mathrm{Ce}\right],[\mathrm{Order}]$ and [Production] are constructed in each period by random numbers limited in the determined interval subject to satisfy corresponding constraints as follows: 


$$
M a_{-} C e=\left(\begin{array}{ll}
0 & 1 \\
1 & 0 \\
1 & 0 \\
0 & 1
\end{array}\right) \quad \longrightarrow \quad M a \_C e=\left(\begin{array}{ll}
0 & 1 \\
0 & 1 \\
1 & 0 \\
0 & 1
\end{array}\right)
$$

Figure 7. The first example for Mutation_Ma_Ca.

$$
M a \_C e=\left(\begin{array}{ll}
0 & 1 \\
1 & 0 \\
1 & 0 \\
0 & 1
\end{array}\right)
$$

$$
M a \_C e=\left(\begin{array}{ll}
1 & 0 \\
0 & 1 \\
1 & 0 \\
0 & 1
\end{array}\right)
$$

Figure 8. The second example for Mutation_Ma_Ca.

(1) The 1st stage assigns the machines to the cells in each period separately concerning the cells size limits.

(2) The 2nd stage determines the orders amount. To obtain a feasible solution more rapidly, first, the total demand of all parts for raw materials in whole periods is calculated and considered as the 1st period order. As a result, other periods' orders are also taken as zero. This method dramatically accelerates the speed to achieve a feasible solution.

(3) In the 3rd stage, one of the parts is selected in one of the periods and the 1st row of matrix Production is formed respect to the vehicle being chosen. If the suitable vacant time position and sufficient time capacity exist on the machines for processing the part, the 1st column including processing machines is filled, otherwise, this part is put aside and another part is chosen. This task is repeated until the capacity of machines in the current period is almost fully utilized. In the same way, the remaining parts are assigned to be manufactured in other periods.

(4) After assigning the parts to the periods, movement times and processing times are calculated and the parts processing priority on the machines is determined.

Concerning the solution structure, the subsequent solutions presented in this section are produced in each iteration by performing a mutation on various components of the current solution.

\subsection{Mechanism for creating a neighboring solution}

To scan through the feasible solution space, it is required to create another feasible solution referred to the neighbor solution via changing the current solution. After that, the solution feasibility has to be checked. If the achieved solution is infeasible, it can be repaired or removed by Repairing or Rejection strategy. Creating a new solution using the current one is done by a mutation operator. In this study, regarding the diversity of the solutions and the variety of the procedures to obtain new solutions, seven mutation operators are utilized.

(1) The intercellular machine movement operator (Mutation_Ma_Ca): this mutation can be carried out in two distinct methods: the 1st one is to transfer a machine from a cell to another cell as illustrated by the example in Figure 7 where machine 2 has been transferred from cell 1 to cell 2 .

In the 2nd method, two machines are substituted in the two cells as shown in Figure 8 where the 1st and 2nd machines are substituted in the cells. 


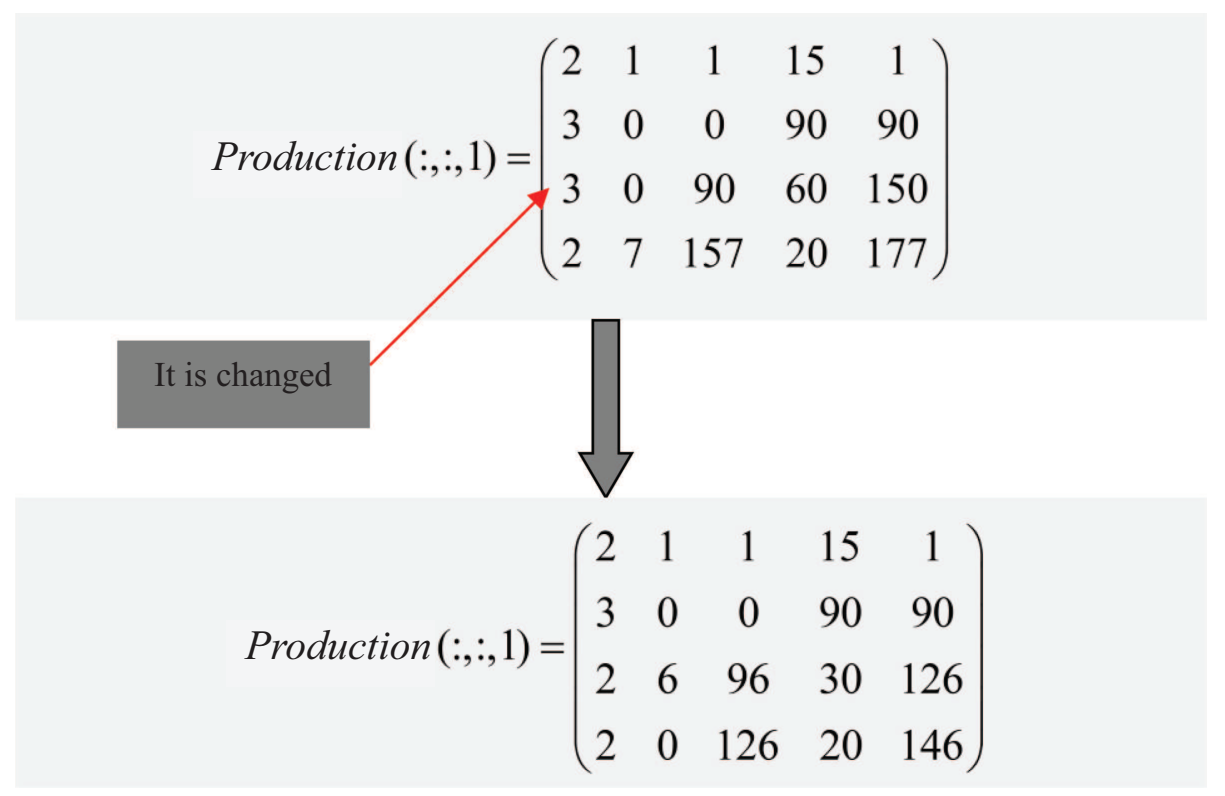

Figure 9. An example of Mutation_Change_Mach.

This mutation is done always through taking the lower and upper limits of the cells into account to lead to a feasible solution and influences components 7-9 in the objective function. Since this alteration can change the intercellular and intracellular movement time, it is required to readjust the starting and finishing times and the movement times in matrix Production. This, in turn, can affect the tardiness time (i.e., component 3 of the objective function).

(2) Order level change operator (Mutation_Order): this mutation transfers the raw material orders from one period to another one by choosing two different periods and transferring each period's order to another period. If the order is transferred from a later period to an earlier one (for instance, from period 4 to period 2), the obtained solution will be feasible again. On the other hand, if this transfer occurs from an earlier period to a later one (e.g., from period 2 to period 3), the raw material should be received on time regarding the lead time to obtain a feasible solution. In the case of the infeasibility of the obtained solution, this solution is rejected by the rejection strategy). This mutation will affect components 5 and 6 in the objective function.

(3) Vehicle change operator (Mutation_Vehicle): this mutation selects one of the periods and its related Production matrices and changes the vehicle used to transport a finished part. To put it simply, it changes the last element in the 1st row in one of the parts of matrix Production. This change may affect Term (4) in the objective function. On the one hand, because the vehicles differ in terms of speed, it can prompt the products to deliver to the markets early or late. As a result, it could influence the tardiness cost in Term (3).

(4) The manufacturing machine change operator (Mutation_Change_Mach): if a part operation can be manufactured by more than one machine and the considered machines have vacant time positions and enough time capacity, a new solution can be achieved via changing the machine which currently manufactures that operation. This can change the movement cost as well as the movement time and completion time of the products; thus it affects the objective function components 3,7 and 8 . To preserve the feasibility of the solution, it is critical to rearrange the manufacturing times of all the parts produced in a period (Fig. 9). 


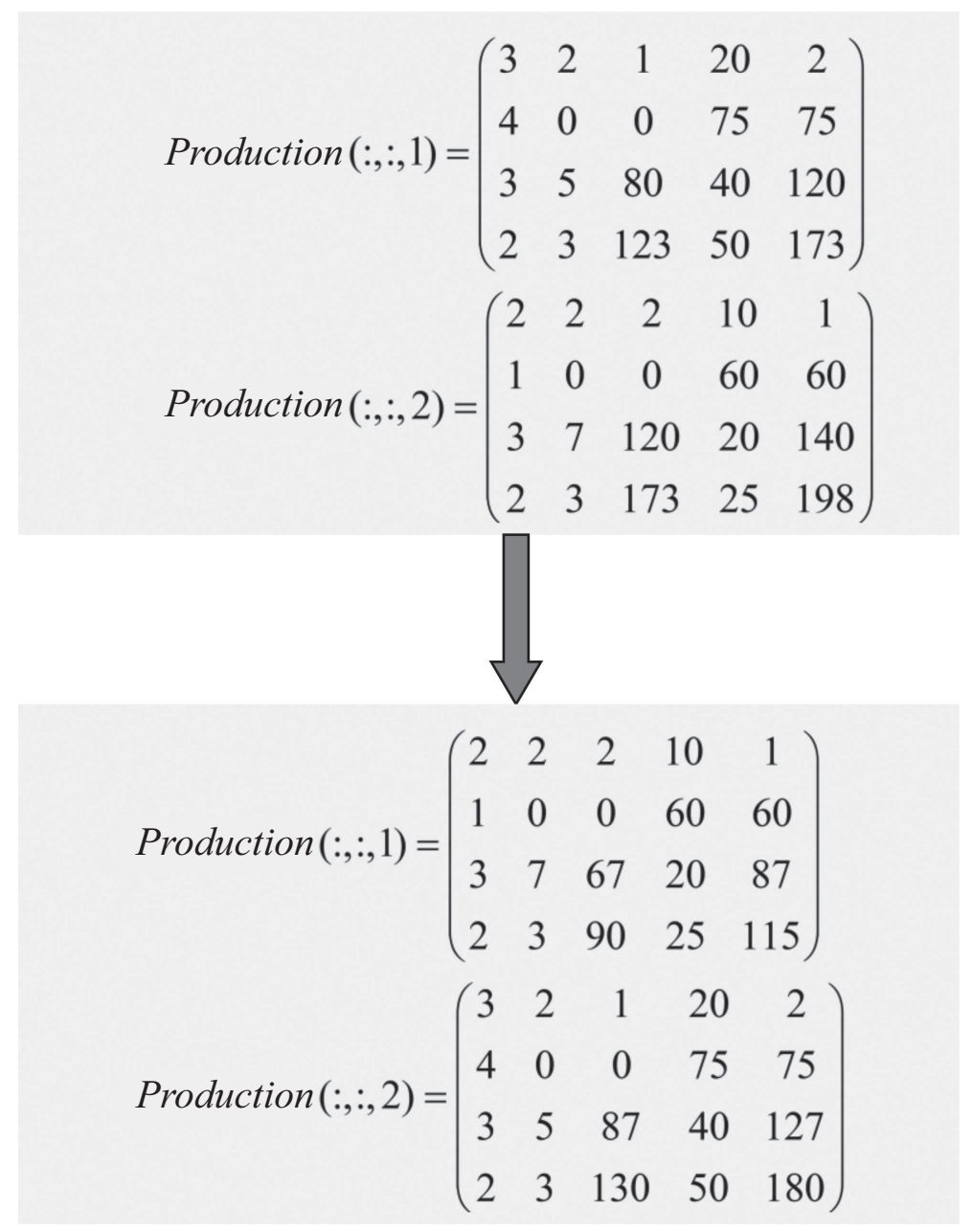

Figure 10. An example of Mutation_Rearrange_Pro.

(5) The parts manufacturing time position change operator (Mutation_Rearrange_Pro): this mutation can cause parts completion times to vary. It only influences Tardiness cost and its resulted solution is always feasible. As seen in the example depicted in Figure 10, in the initial solution, part 3 completion time is 173 and part 2 completion time is 198 . As the manufacturing time position varies by implementing this mutation, part 3 completion time is changed to 180 and part 2 completion time is changed to 115. Although part 3 completion time in the new solution has increased 7 units, part 2 completion time has decreased 83 units.

(6) The parts production periods change operator (Mutation_Change_Pro): producing a part earlier than its due date incurs inventory holding cost while producing later than its due date burdens tardiness costs. This mutation provides the ground for an effective approach to decrease these costs to produce the parts in the same period when the demands happen. The mutation selects the production period of a part randomly and transfers it to another period. If in the destination period, there is not enough time for processing, the solution is infeasible and is rejected. In the next stage, if the origin period is earlier than the destination period, the solution feasibility is guaranteed. However, if the origin period is later than the destination period, the solution might become infeasible. Therefore, Repairing strategy strives to rearrange the orders to make the solution feasible. In the end, the starting and ending times of manufacturing parts are 
TABLE 1. Manufacturing times data for illustrative example.

\begin{tabular}{llllllllll}
\hline \hline & \multicolumn{3}{c}{$k_{1}$} & \multicolumn{1}{c}{$k_{2}$} & & & $k_{3}$ \\
\cline { 2 - 10 } & $M_{1}$ & $M_{2}$ & $M_{3}$ & $M_{1}$ & $M_{2}$ & $M_{3}$ & $M_{1}$ & $M_{2}$ & $M_{3}$ \\
\hline$P_{1}$ & 10 & 0 & 0 & 0 & 0 & 8 & 20 & 0 & 0 \\
$P_{2}$ & 0 & 0 & 6 & 0 & 0 & 8 & 0 & 5 & 0 \\
$P_{3}$ & 0 & 0 & 6 & 0 & 8 & 8 & 0 & 30 & 0 \\
\hline
\end{tabular}

TABle 2. Data for the machines time capacity, costs of the machines relocation, the raw materials ordering and the raw materials inventory holding for illustrative example.

\begin{tabular}{ccccccccccc}
\hline \hline & \multicolumn{3}{c}{$\mathrm{TM}_{m h}$} & \multicolumn{3}{c}{$u_{m h}$} & \multicolumn{3}{c}{$\sigma_{r h}$} & \multicolumn{2}{c}{$\mu_{r h}$} \\
\cline { 2 - 10 } & $M_{1}$ & $M_{2}$ & $M_{3}$ & $M_{1}$ & $M_{2}$ & $M_{3}$ & $r_{1}$ & $r_{2}$ & $r_{1}$ & $r_{2}$ \\
\hline$h_{1}$ & 650 & 600 & 550 & 70 & 65 & 80 & 3 & 2 & 1 & 2 \\
$h_{2}$ & 650 & 600 & 550 & 70 & 65 & 80 & 4 & 3 & 1 & 2 \\
\hline
\end{tabular}

rearranged in the destination period. This mutation can be effective on the objective function components $1-8$.

(7) Two parts substitution between two periods operator (Mutation_Swap_Prod): this mutation is similar to mutation No. 6 in performance except that here a part is transferred from the origin to the destination period and in return, another part is transferred from the destination to the origin period. By applying this mutation, regarding the changes in time positions and the manufacturing times in both periods, there is more probability for generating infeasible solutions compared to mutation No. 6. If the obtained solution is infeasible, it is rejected, otherwise, like the previous state, the related orders and also the manufacturing times are rearranged. Similarly, this mutation can influence the objective function components 1-8.

The pseudo code of the developed SA for the proposed model, termination condition, acceptance/rejection mechanism of a neighborhood solution and cooling schedule including initial temperature, Markov chain length (MCL), and cooling rate are defined as same as those defined by Kia et al. [26].

\section{Computational Results}

\subsection{An illustrative numerical example}

To validate the integrated model and illustrate its various features, an illustrative numerical example is solved and analyzed by CPLEX software. In this small-sized example, 3 parts, 3 machines, 2 cells, 2 vehicles, 2 markets, 2 raw materials and 2 periods have been taken into account. The upper and lower cell size limits are 2 and 1 , respectively. The intra-cell movement time and inter-cell movement times for all parts are 3 and 6 , respectively. Also, the inter-cell movement costs are 0.6 and 1.5 and the intra-cell movement costs as 0.2 and 0.3 , for all the parts in periods 1 and 2, respectively. The lead times for raw materials 1 and 2 are 2 and 1, respectively. The reason for defining such a small example is to make it possible to track material flows and explain the relations between the integrated decisions more straightforward.

Table 1 illustrates the manufacturing times for each type of parts on various machines. For instance, the second operation of part 3 can be manufactured on either machine 2 or 3 with 8 -time units.

Table 2 lists the data for the machines time capacity, the machines relocation costs, the raw materials ordering costs and the raw materials inventory holding costs. For example, the time capacity for machine 2 in period 2 is $600 \mathrm{~h}$ and raw materials type 1 ordering cost in period 2 is 4 units. 
TABLE 3. Raw materials initial inventory level and the usage coefficients for illustrative example.

\begin{tabular}{lllllll}
\hline \hline & \multicolumn{3}{c}{$\omega_{p r}$} & \multicolumn{3}{c}{$\mathrm{IS}_{p r}$} \\
\cline { 2 - 7 } & $P_{1}$ & $P_{2}$ & $P_{3}$ & $P_{1}$ & $P_{2}$ & $P_{3}$ \\
\hline$r_{1}$ & 1 & 0 & 1 & 30 & 0 & 30 \\
$r_{2}$ & 4 & 4 & 0 & 70 & 40 & 0 \\
\hline
\end{tabular}

TABLE 4. Parts transportation costs and times for illustrative example.

\begin{tabular}{llllll}
\hline \hline & & \multicolumn{2}{c}{$i_{1}$} & \multicolumn{2}{c}{$i_{2}$} \\
\cline { 3 - 6 } & & $h_{1}$ & $h_{2}$ & $h_{1}$ & $h_{2}$ \\
\hline \multirow{2}{*}{$\phi_{i j h}$} & $j_{1}$ & 700 & 700 & 200 & 200 \\
& $j_{2}$ & 1000 & 1300 & 400 & 400 \\
$f_{i j}$ & $j_{1}$ & 30 & & 100 & \\
& $j_{2}$ & 50 & & 200 & \\
\hline
\end{tabular}

TABLE 5. Other input parameters values for illustrative example.

\begin{tabular}{cccccccccccc}
\hline \hline & & \multicolumn{3}{c}{$\beta_{p j h}$} & \multicolumn{2}{c}{$D_{p j h}$} & \multicolumn{2}{c}{$d u_{p j h}$} & \multicolumn{2}{c}{$\pi_{p j h}$} & \multicolumn{2}{c}{$g_{p j h}^{\prime}$} \\
\cline { 3 - 12 } & $h_{1}$ & $h_{2}$ & $h_{1}$ & $h_{2}$ & $h_{1}$ & $h_{2}$ & $h_{1}$ & $h_{2}$ & $h_{1}$ & $h_{2}$ \\
\hline \multirow{2}{*}{$j_{1}$} & $P_{1}$ & 2 & 3 & 0 & 0 & 0 & 0 & 800 & 800 & 1000 & 1000 \\
& $P_{2}$ & 3 & 4 & 10 & 0 & 350 & 0 & 900 & 900 & 1010 & 1010 \\
& $P_{3}$ & 1 & 2 & 0 & 0 & 0 & 0 & 1000 & 1000 & 950 & 950 \\
$j_{2}$ & $P_{1}$ & 3 & 3 & 20 & 0 & 530 & 0 & 700 & 700 & 900 & 900 \\
& $P_{2}$ & 3 & 3 & 0 & 0 & 0 & 0 & 850 & 850 & 950 & 950 \\
& $P_{3}$ & 3 & 3 & 0 & 20 & 0 & 890 & 900 & 900 & 900 & 900 \\
\hline
\end{tabular}

Table 3 includes the data about the raw materials initial inventory levels at the beginning of period 1 as well as the usage coefficients of the raw materials. For instance, part 1 needs raw material types 1 and 2 with usage coefficients 1 and 4, respectively. Also, at the beginning of period 1, 40 units of the raw material type 2 are available in the plant warehouse for manufacturing part 2 .

Table 4 represents the parts transportation costs and times from the plant to the markets by diverse vehicles. For instance, the parts transportation cost and time from the plant to market 2 using vehicle type 2 during period 2 are 400 and 200, respectively. Since the speed of type 1 vehicle is higher than that of vehicle 2 , thus it has a higher cost and lower time.

In Table 5, other input parameters values including tardiness penalty cost, demand levels, due dates, parts inventory holding costs in the markets warehouse and the backorders costs are given.

Figure 11 schematically illustrate the integrated DCMS problem, as well as the interrelated decisions of different layers including MRP, scheduling, PP and TP decisions.

In the first layer of the considered supply chain, we integrate MRP for raw materials received from different suppliers with production planning for the plant. The demand of a market in a period has to be satisfied using the internal production in one of the periods that may be one or more periods earlier than the intended demand period as inventory holding strategy, one or more periods later than the intended demand period as backorders strategy and or in the same period. As it has been discussed in the literature of production systems, MRP and Master Production Schedule (MPS) are strongly interconnected in a way that the decisions that are made for 


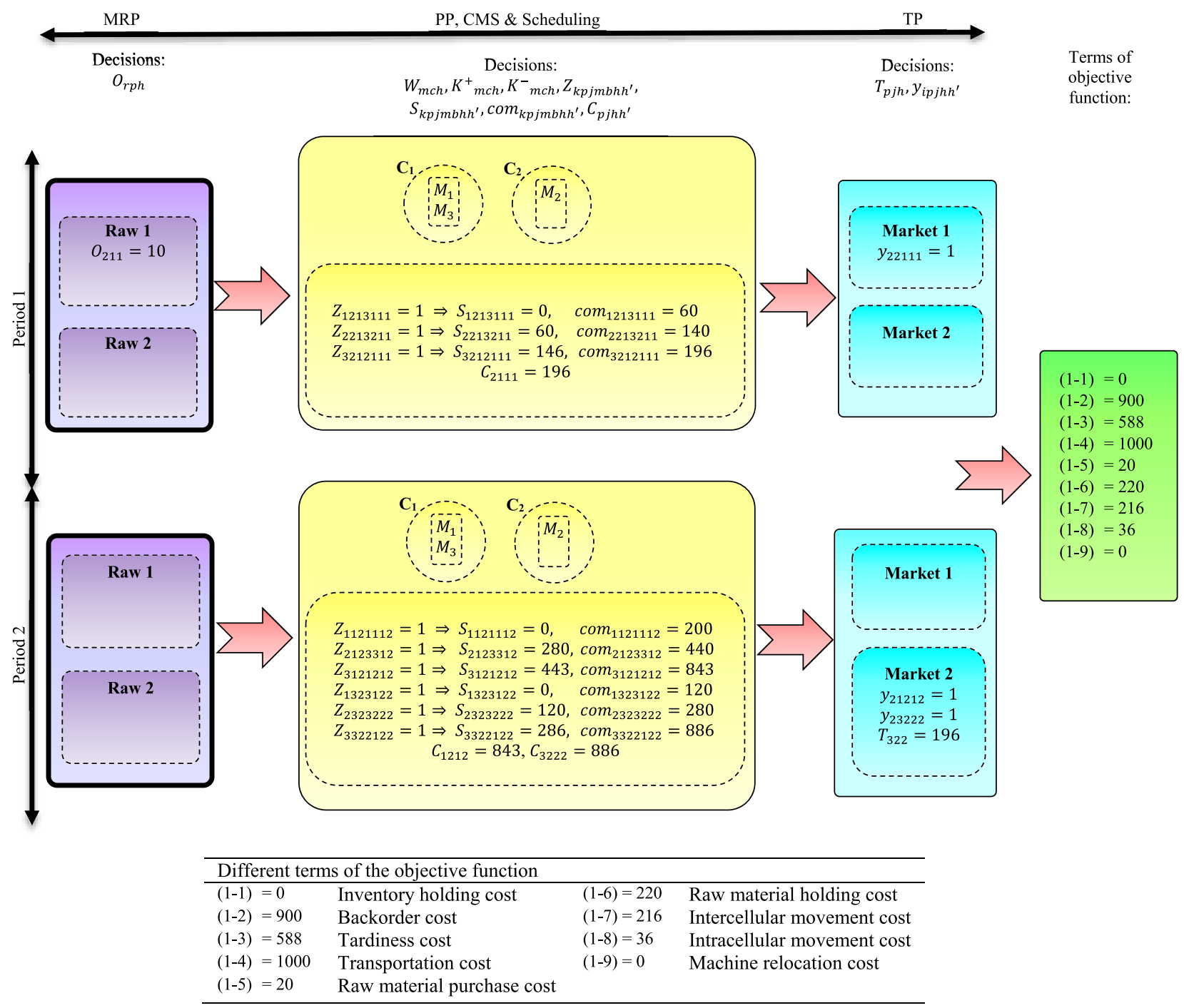

Figure 11. Illustration of the proposed DCMS problem.

production period would affect on the order release period of raw materials. This justifies the integration of MRP and PP decisions in our model.

From the input data explained above and the results reported in Figure 11, it can be seen that to provide the demands of markets 1 and 2 , we need $(1 \times 20=20),(0 \times 10=0)$, and $(1 \times 20=20)$ number of raw material type 1 for each products $1-3$, and $(4 \times 20=80),(4 \times 10=40)$, and $(0 \times 20=0)$ number of raw material type 2 for each products $1-3$, respectively. Checking the initial stock of raw materials in the warehouse, we will see that we have enough stock for all the demands except initial stock of raw materials 2 for product 1 . $\mathrm{IS}_{12}$ is 70 ; however, the required amount is 80 units. Thus, it is needed to order 10 units, $O_{211}=10$. This requirement is needed to be provided in the first period, and this is while the lead time for raw materials 2 is 1 . Even if we order this raw material in period 1, it will be supplied in period 2. So, it means that in providing the demand 


\begin{tabular}{|c|c|c|c|c|}
\hline Part & Transportation vehicle & $\begin{array}{l}\text { Transportation } \\
\text { cost }\end{array}$ & Tardiness cost & Total \\
\hline & 1 & 1300 & $0 \leftarrow($ Backorder cost $)$ & 1300 \\
\hline & 2 & 400 & $0 \leftarrow($ Backorder cost $)$ & 400 \\
\hline & 1 & 700 & $0 \leftarrow[(196+30) \leq 350]$ & 700 \\
\hline & & 200 & $0 \leftarrow[(196+100) \leq 350]$ & 200 \\
\hline & 1 & 1300 & $46 \leftarrow[(886+50)-890=46]$ & 1346 \\
\hline & 2 & 400 & $196 \leftarrow[(886+200)-890=196]$ & 596 \\
\hline
\end{tabular}

FiguRE 12. Different transportation vehicles and different costs for each method.

for product 1 in market 2 , we will face some backorder costs. The backorder cost of product 1 of market 2 in period 1 is 900 , objective function (3.1b).

As the considered vehicle fleet is heterogeneous, the optimal selection of vehicles based on transportation times and cost are performed. In one hand, selecting slow vehicles with low transportation costs may result from tardiness in delivery, on the other hand, selecting fast vehicles with high transportation costs may remove tardiness cost but at higher transportation cost.

In this example, products transportation decisions have to be made according to two vehicles. Generally, faster vehicles of type 1 with higher transportation costs and shorter delivery times deliver products to markets earlier than due dates, which results in fewer tardiness costs. Therefore, to select a vehicle, a trade-off between transportation cost and tardiness cost is made via an objective function. Taking into account the tardiness cost at the market warehouse, the model decides between two vehicles.

Figure 12 shows different vehicles and the costs for each vehicle. By selecting vehicle 1, total costs of transportation $(1300+700+1300)$ and tardiness $(0+0+46)$ will be $(3300+46=3346)$. Similarly, by choosing vehicle 2 , we will have $400+200+400=1000$ transportation cost and 196 tardiness cost, in total 1196. From Figure 12, it is understood that the model will choose vehicle 2, resulting in some savings in transportation cost equal to $(3300-1000=2300)$, but some lost in tardiness cost $(196-46=150)$, totally more savings equal to $2300-150=2150$.

Figure 13 depicts cells configuration, parts scheduling, inter- and intra-cell movements and delivery time to markets during 2 periods.

In the proposed model, we incorporate the part scheduling decisions as to the final detailed stage of production planning in a CMS. The scheduling of parts in a CMS is affected by PP decisions during each period as scheduling during a period is released only for parts that have been decided to be produced in that period. Therefore, the amount of production as an output of PP is a fundamental input for scheduling phase. This establishes the dependency of parts scheduling to PP decisions. For example, the demand for part 1 is fulfilled with one period delay due to a shortage in raw materials, thus, we face some backorder costs. As production of part 1 is postponed to period 2, its operations scheduling is developed in period 2 as well. As seen in Figure 13, parts 2 and 3 are decided to be produced in their demand period as a PP strategy, so scheduling of parts 2 and 3 is provided at the same demand period

In the second layer of the considered supply chain connecting a plant with a CMS concept to the markets, we also integrate the decisions of PP and transportation planning in a detailed scheduling level. In other words, 


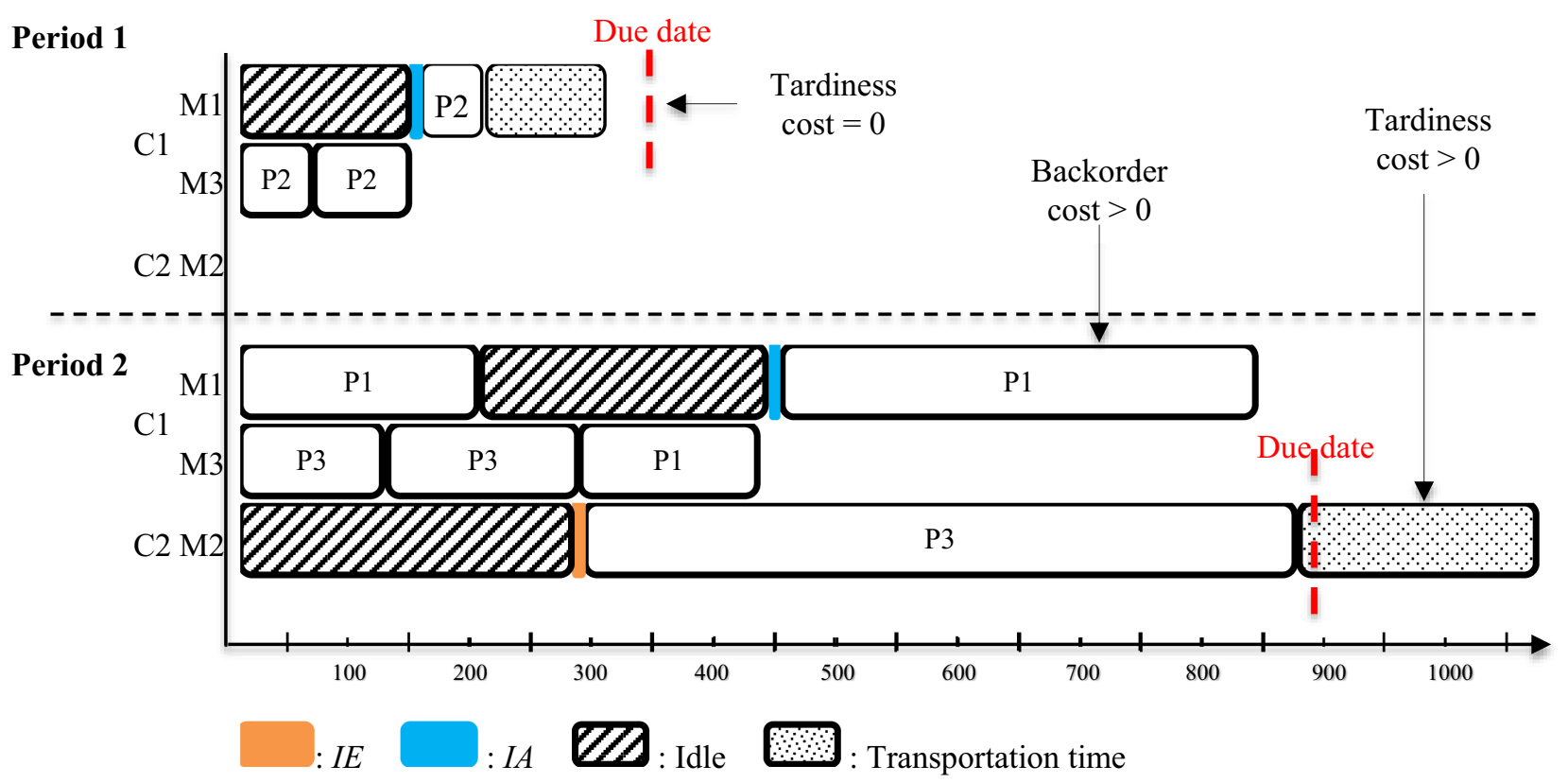

FiguRE 13. Cells configuration and parts scheduling during the periods.

the part scheduling is connected to the delivery time of vehicles to markets. By synchronizing the parts completion times and the vehicle departure times, we can determine the exact arrival times of the selected vehicles to each market. Reaching to the markets after the due date given for each part results in a tardiness and burden a relevant penalty. Therefore, by having scheduling decisions, we can deliver parts to the markets in appropriate time to minimize any tardiness.

For example, part 2 is delivered to market 1 before its due date in period 1 , hence, there is no tardiness penalty for part 2. However, according to the coordinated scheduling for part 3 and its completion time, as it is delivered to market 2 after its due date, we face some tardiness costs.

From the above illustrative test problem, it can be inferred that all the decisions in different layers are interconnected to each other and the way we approach the problem makes the decisions of different layers altogether in a comprehensive fashion. It is well-reasoned that the decisions about satisfying the market demand in different periods are interconnected to the decisions of producing the products, as well as transportation planning. Obviously, according to production planning, a plant needs to decide about its PP decisions, cell configuration, and CMS decisions, and parts scheduling. By considering that all of these decisions are affected by upper-layer decisions, which provide the raw materials by the suppliers (MRP), we recognize why in this problem different layer decisions are included to our mathematical formulation comprehensively.

In the next section, to have a better managerial intuition about the influence of different parameters and decisions of different layers on each other, a sensitivity analysis is carried out. Also, we integrate PP and dynamic cell formation decisions. The necessity of this integration are addressed by many researchers, as cited in Section 2.2. As a brief explanation, main decision of PP is determining the amount of production in each period. Therefore, the required production capacity in each cell, which is obtained based on the production amount assigned to that cell, determines the required number of machines. Next, knowing the number of machines assigned to each cell is a crucial decision for a cell formation problem. For instance, in our example, machines 1 and 3 are assigned to cell 1 and remaining machine 2 is assigned to the cell. By this allocation of machines in 2 cells, we have 3 intra-cell movements and only one inter-cell movement. This assignment remains unchanged for the second period to prevent any machine relocation cost. Nevertheless, as in multi-period production planning, 
TABle 6. Manufacturing times data for Sensitivity analysis example.

\begin{tabular}{|c|c|c|c|c|c|c|c|c|c|c|c|c|c|c|c|}
\hline & \multicolumn{5}{|c|}{$k_{1}$} & \multicolumn{5}{|c|}{$k_{2}$} & \multicolumn{5}{|c|}{$k_{3}$} \\
\hline & $M_{1}$ & $M_{2}$ & $M_{3}$ & $M_{4}$ & $M_{5}$ & $M_{1}$ & $M_{2}$ & $M_{3}$ & $M_{4}$ & $M_{5}$ & $M_{1}$ & $M_{2}$ & $M_{3}$ & $M_{4}$ & $M_{5}$ \\
\hline$P_{1}$ & 10 & 0 & 0 & 0 & 0 & 0 & 0 & 8 & 0 & 25 & 20 & 0 & 0 & 0 & 7 \\
\hline$P_{2}$ & 0 & 0 & 6 & 0 & 20 & 0 & 0 & 0 & 0 & 9 & 0 & 5 & 0 & 0 & 0 \\
\hline$P_{3}$ & 0 & 0 & 0 & 8 & 0 & 0 & 8 & 0 & 15 & 0 & 0 & 30 & 0 & 8 & 0 \\
\hline$P_{4}$ & 0 & 10 & 0 & 50 & 0 & 0 & 30 & 0 & 6 & 0 & 0 & 10 & 0 & 0 & 0 \\
\hline$P_{5}$ & 60 & 0 & 0 & 0 & 10 & 9 & 0 & 0 & 0 & 20 & 0 & 0 & 6 & 0 & 0 \\
\hline
\end{tabular}

TABLE 7. Data for the machines time capacity, costs of the machines relocation, the raw material ordering and the raw materials inventory holding for Sensitivity analysis example.

\begin{tabular}{|c|c|c|c|c|c|c|c|c|c|c|c|c|c|c|c|c|}
\hline & \multicolumn{5}{|c|}{$\mathrm{TM}_{m h}$} & \multicolumn{5}{|c|}{$u_{m h}$} & \multicolumn{3}{|c|}{$\sigma_{r h}$} & \multicolumn{3}{|c|}{$\mu_{r h}$} \\
\hline & $M_{1}$ & $M_{2}$ & $M_{3}$ & $M_{4}$ & $M_{5}$ & $M_{1}$ & $M_{2}$ & $M_{3}$ & $M_{4}$ & $M_{5}$ & $r_{1}$ & $r_{2}$ & $r_{3}$ & $r_{1}$ & $r_{2}$ & $r_{3}$ \\
\hline$h_{1}$ & 700 & 550 & 550 & 450 & 240 & 70 & 60 & 80 & 80 & 60 & 3 & 2 & 1 & 1 & 2 & 2 \\
\hline$h_{2}$ & 650 & 600 & 600 & 700 & 340 & 75 & 65 & 85 & 80 & 65 & 2 & 1 & 4 & 2 & 3 & 2 \\
\hline$h_{3}$ & 750 & 550 & 600 & 400 & 360 & 80 & 70 & 85 & 85 & 70 & 2 & 4 & 1 & 3 & 4 & 3 \\
\hline$h_{4}$ & 500 & 500 & 500 & 500 & 300 & 85 & 80 & 90 & 85 & 75 & 3 & 5 & 3 & 4 & 5 & 4 \\
\hline
\end{tabular}

TABLE 8. Raw materials initial inventory level and the usage coefficients for Sensitivity analysis example.

\begin{tabular}{rllllllrrrr}
\hline \hline & \multicolumn{9}{c}{$\omega_{p r}$} & \multicolumn{6}{c}{$\mathrm{IS}_{p r}$} \\
\cline { 2 - 11 } & $P_{1}$ & $P_{2}$ & $P_{3}$ & $P_{4}$ & $P_{5}$ & $P_{1}$ & $P_{2}$ & $P_{3}$ & $P_{4}$ & $P_{5}$ \\
\hline$r_{1}$ & 1 & 0 & 1 & 2 & 0 & 30 & 0 & 30 & 82 & 0 \\
$r_{2}$ & 4 & 4 & 0 & 0 & 2 & 70 & 40 & 0 & 0 & 32 \\
$r_{3}$ & 2 & 0 & 2 & 3 & 1 & 15 & 0 & 15 & 38 & 15 \\
\hline
\end{tabular}

the amount of production is changing to balance the production capacity with fluctuating demand, so machines reallocation may be inevitable to reduce material handling costs. In conclusion, PP and cells reconfiguration decisions are strongly interrelated.

\subsection{Sensitivity analysis}

In this section, to evaluate the effect of some parameters on the model performance, a sensitivity analysis is carried out by changing the initial values of the parameters and measuring the resulted changes in the objective function value. In this state, a problem with 5 parts, 5 machines, 2 cells, 3 vehicles, 2 markets, 3 raw materials and 4 periods is considered. The upper and lower cell size limits are 4 and 2 , respectively. The intercell movement times for parts 1-5 are 6,6,6,8 and 9, respectively and intracellular movement time as $3,4,3$, 5 and 4, respectively. Also, the inter-cell movement costs are 0.6, 0.7, 0.9 and 1 and the intra-cell movement costs as $0.2,0.3,0.4$ and 0.5 , for periods $1-4$, respectively. The lead times for raw materials $1-3$ are 2,1 and 1 , respectively. Tables $6-10$ present the input data with a similar explanation given for the illustrative example data.

Table 11 displays how different raw materials are ordered during 4 periods. For instance, 13 units of raw materials type 1 are ordered in period 1 to produce part type 1 . Because type 1 raw materials lead time is 2 , then this raw material reaches the plant within two next periods (i.e., period 3).

Figure 14 depicts machines allocation to cells and parts scheduling inside the plant during 4 periods. 
TABLE 9. Parts transportation costs and times for Sensitivity analysis example.

\begin{tabular}{|c|c|c|c|c|c|c|c|c|c|c|c|c|c|}
\hline & & \multicolumn{4}{|c|}{$i_{1}$} & \multicolumn{4}{|c|}{$i_{2}$} & \multicolumn{4}{|c|}{$i_{3}$} \\
\hline & & $h_{1}$ & $h_{2}$ & $h_{3}$ & $h_{4}$ & $h_{1}$ & $h_{2}$ & $h_{3}$ & $h_{4}$ & $h_{1}$ & $h_{2}$ & $h_{3}$ & $h_{4}$ \\
\hline \multirow[t]{2}{*}{$\phi_{i j h}$} & $j_{1}$ & 500 & 550 & 560 & 570 & 100 & 110 & 130 & 150 & 50 & 50 & 65 & 80 \\
\hline & $j_{2}$ & 800 & 830 & 850 & 880 & 250 & 260 & 280 & 290 & 70 & 85 & 85 & 90 \\
\hline \multirow[t]{2}{*}{$f_{i j}$} & $j_{1}$ & \multicolumn{4}{|c|}{40} & \multicolumn{4}{|c|}{150} & \multicolumn{4}{|c|}{300} \\
\hline & $j_{2}$ & \multicolumn{4}{|c|}{100} & \multicolumn{4}{|c|}{200} & \multicolumn{4}{|c|}{400} \\
\hline
\end{tabular}

TABLE 10. Other input parameters values for Sensitivity analysis example.

\begin{tabular}{|c|c|c|c|c|c|c|c|c|c|c|c|c|c|c|c|c|c|c|c|c|c|}
\hline & & \multicolumn{4}{|c|}{$\beta_{p j h}$} & \multicolumn{4}{|c|}{$D_{p j h}$} & \multicolumn{4}{|c|}{$d u_{p j h}$} & \multicolumn{4}{|c|}{$\pi_{p j h}$} & \multicolumn{4}{|c|}{$g_{p j h}^{\prime}$} \\
\hline & & $h_{1}$ & $h_{2}$ & $h_{3}$ & $h_{4}$ & $h_{1}$ & $h_{2}$ & $h_{3}$ & $h_{4}$ & $h_{1}$ & $h_{2}$ & $h_{3}$ & $h_{4}$ & $h_{1}$ & $h_{2}$ & $h_{3}$ & $h_{4}$ & $h_{1}$ & $h_{2}$ & $h_{3}$ & $h_{4}$ \\
\hline \multirow[t]{5}{*}{$j_{1}$} & $P_{1}$ & 2 & 3 & 3 & 4 & 0 & 0 & 23 & 10 & 0 & 0 & 650 & 400 & 900 & 910 & 920 & 930 & 1060 & 1070 & 1090 & 1090 \\
\hline & $P_{2}$ & 3 & 4 & 4 & 5 & 10 & 0 & 0 & 0 & 350 & 0 & 0 & 0 & 910 & 920 & 930 & 950 & 1010 & 1030 & 1050 & 1060 \\
\hline & $P_{3}$ & 1 & 2 & 3 & 4 & 0 & 0 & 0 & 15 & 0 & 0 & 0 & 150 & 1000 & 1300 & 1400 & 1500 & 940 & 970 & 980 & 990 \\
\hline & $P_{4}$ & 4 & 4 & 5 & 5 & 12 & 0 & 15 & 0 & 310 & 0 & 450 & 0 & 750 & 780 & 790 & 830 & 870 & 880 & 910 & 930 \\
\hline & $P_{5}$ & 2 & 3 & 4 & 4 & 0 & 0 & 0 & 0 & 0 & 0 & 0 & 0 & 700 & 740 & 760 & 780 & 800 & 850 & 880 & 890 \\
\hline \multirow[t]{5}{*}{$j_{2}$} & $P_{1}$ & 3 & 3 & 4 & 4 & 20 & 0 & 0 & 0 & 530 & 0 & 0 & 0 & 820 & 850 & 860 & 870 & 900 & 930 & 940 & 950 \\
\hline & $P_{2}$ & 3 & 3 & 4 & 4 & 0 & 0 & 0 & 15 & 0 & 0 & 0 & 490 & 800 & 830 & 840 & 850 & 950 & 970 & 990 & 1000 \\
\hline & $P_{3}$ & 3 & 3 & 4 & 5 & 0 & 20 & 0 & 0 & 0 & 890 & 0 & 0 & 850 & 860 & 880 & 890 & 900 & 910 & 930 & 950 \\
\hline & $P_{4}$ & 3 & 3 & 4 & 5 & 0 & 20 & 0 & 0 & 0 & 820 & 0 & 0 & 800 & 850 & 900 & 920 & 920 & 940 & 980 & 1000 \\
\hline & $P_{5}$ & 3 & 4 & 5 & 5 & 15 & 0 & 15 & 0 & 690 & 0 & 560 & 0 & 850 & 870 & 890 & 900 & 980 & 990 & 1000 & 1010 \\
\hline
\end{tabular}

TABLE 11. Raw materials orders.

\begin{tabular}{|c|c|c|c|c|c|c|c|c|c|c|c|c|}
\hline & \multicolumn{4}{|c|}{$r_{1}$} & \multicolumn{4}{|c|}{$r_{2}$} & \multicolumn{4}{|c|}{$r_{3}$} \\
\hline & $h_{1}$ & $h_{2}$ & $h_{3}$ & $h_{4}$ & $h_{1}$ & $h_{2}$ & $h_{3}$ & $h_{4}$ & $h_{1}$ & $h_{2}$ & $h_{3}$ & $h_{4}$ \\
\hline$p_{1}$ & 13 & 10 & & & 10 & 92 & 40 & & 71 & & 20 & \\
\hline$p_{2}$ & & & & & & & 60 & & & & & \\
\hline$p_{3}$ & 5 & & & & & & & & 55 & & & \\
\hline$p_{4}$ & 12 & & & & & & & & 103 & & & \\
\hline$p_{5}$ & & & & & 28 & & & & 15 & & & \\
\hline
\end{tabular}

Figure 15 indicates the parts transportation plan from the plant to the markets during 4 periods. For example, the demand of part 1 by market 2 in period 1 equal to 20 units is supplied by the plant in period 2 and transported via vehicle type 3 .

Table 12 represents the tardiness values for different parts in different periods. For instance, part 1 demanded by market 1 during period 3 delays 81 -time units.

Different parameters including the due date required vehicle time, inventory holding cost, backorder cost, order cost, relocation cost, intra and intercellular movement time are increased by $10 \%-40 \%$. We also investigate the effect of increasing the lead times based on different increasing scenarios. It is worth noting that the initial lead times for raw materials 1-3 are 2, 1 and 1, respectively (let's call it scenario $(2,1,1)$ ), and the new increased lead times scenarios are $(3,1,1),(2,2,1)$, and $(2,1,2)$. The influences of such variations over the results are presented through Figures 16-22.

In some cases, the influence of the parameter increment is expectable; however, in some other cases, the effect of the changes is far from expectation. This unexpected behavior confirms the importance and virtue of such a comprehensive formulation. The model detects a better solution with a lower objective function value, in which 


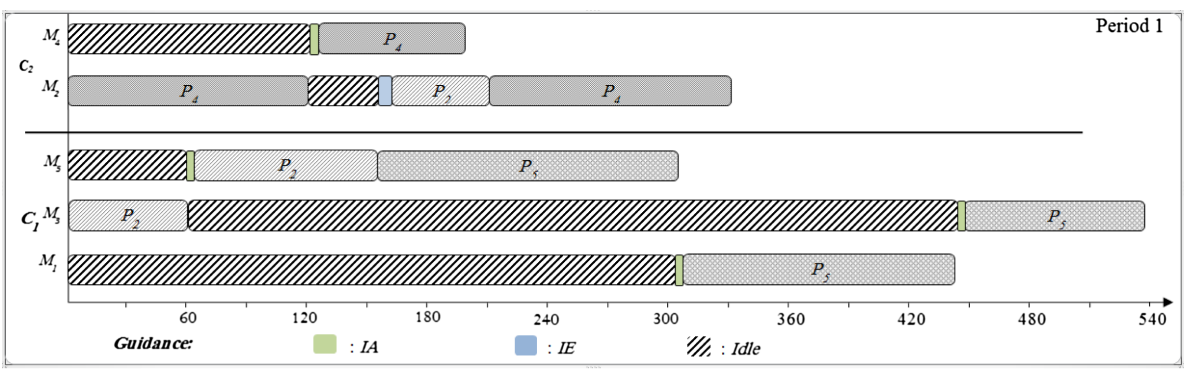

\section{a. Period 1}

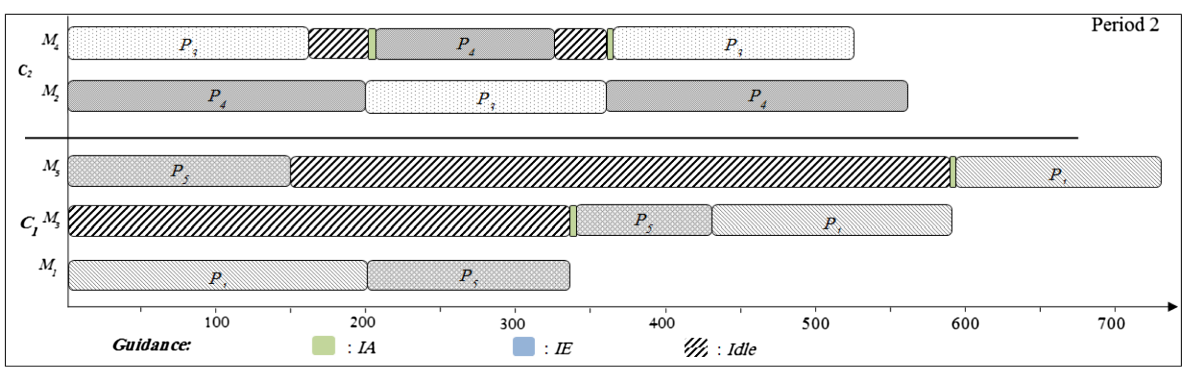

\section{b. Period 2}

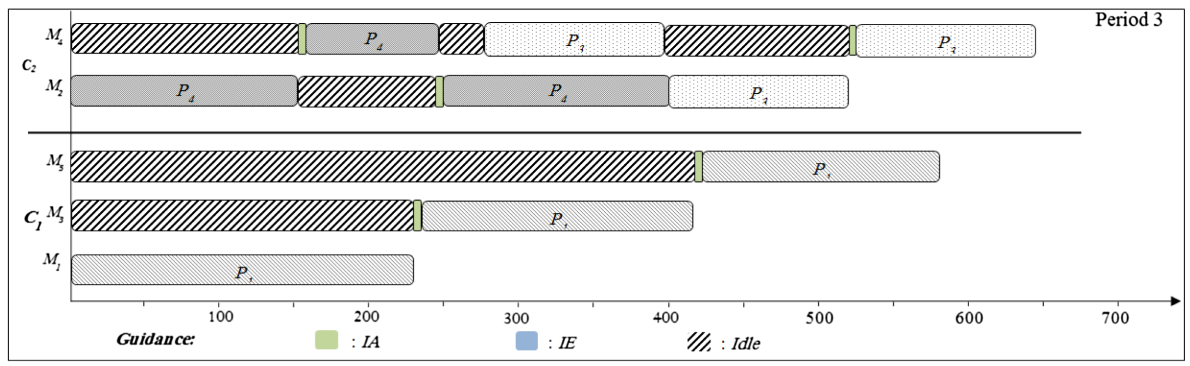

\section{c. Period 3}

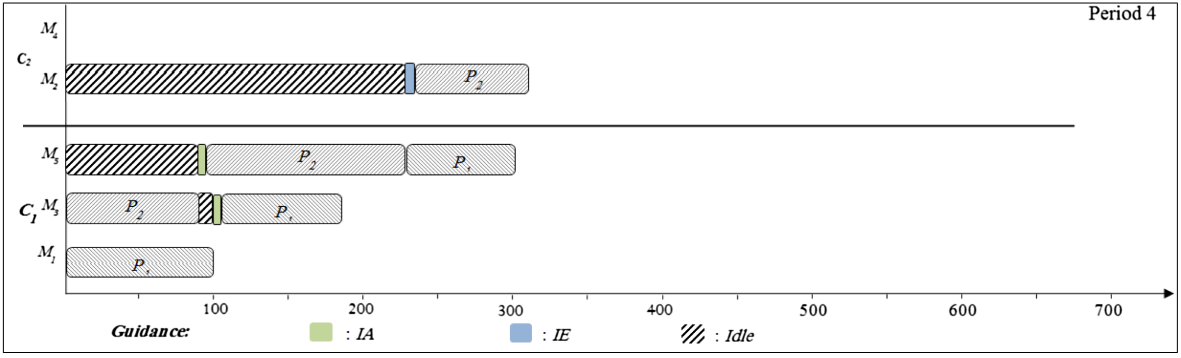

\section{d. Period 4}

FIgURE 14. Cells configuration and parts scheduling during four periods.

the offered solution is far from our rough expectations. For example, increasing the due dates, it seems logical that the trend according to the total objective function should be descending as less tardiness is expected to happen, and the results confirm this expectancy. Opposing behaviors of different terms in the objective function, finally, come up with a solution with a lower objective function value. On the other hand, increasing the due dates, one may expect that the tardiness cost shows a descending trend, this is while in some cases, it shows increments. The reason of such a behavior is that the model previously decided to produce the part in precedent 


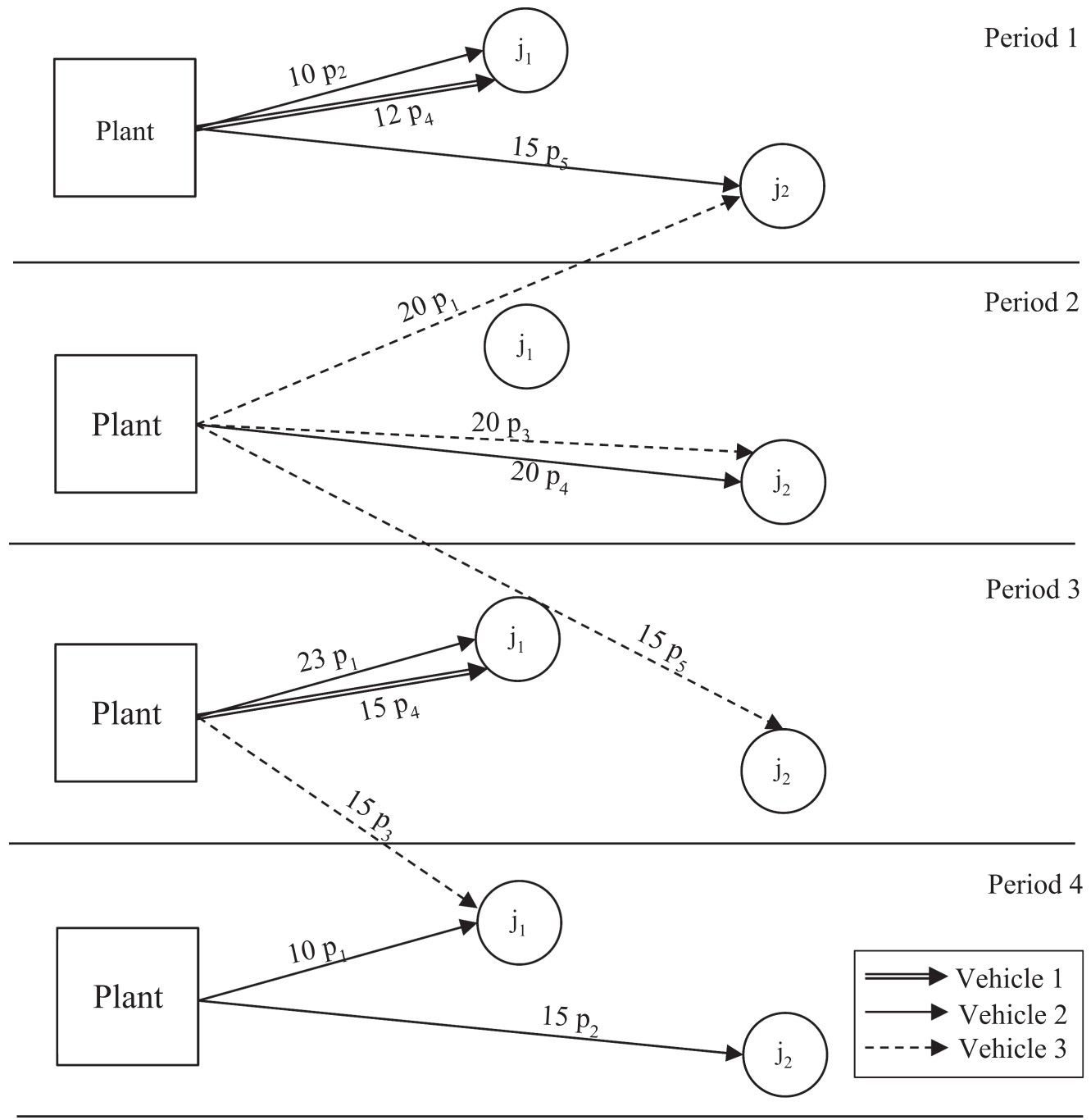

FiguRE 15. Transportation plans for parts from the plant to the markets during four periods.

TABLE 12. Tardiness values.

\begin{tabular}{lcccccccc}
\hline \hline & \multicolumn{3}{c}{$j_{1}$} & \multicolumn{5}{c}{$j_{1}$} \\
\cline { 2 - 7 } & $h_{1}$ & $h_{2}$ & $h_{3}$ & $h_{4}$ & $h_{1}$ & $h_{2}$ & $h_{3}$ & $h_{4}$ \\
\hline$p_{1}$ & & & 81 & 49 & & & & \\
$p_{2}$ & 10 & & & & & & & 20 \\
$p_{3}$ & & & & & & 33 & & \\
$p_{4}$ & 50 & & & & & & & \\
$p_{5}$ & & & & & 47 & & \\
\hline
\end{tabular}



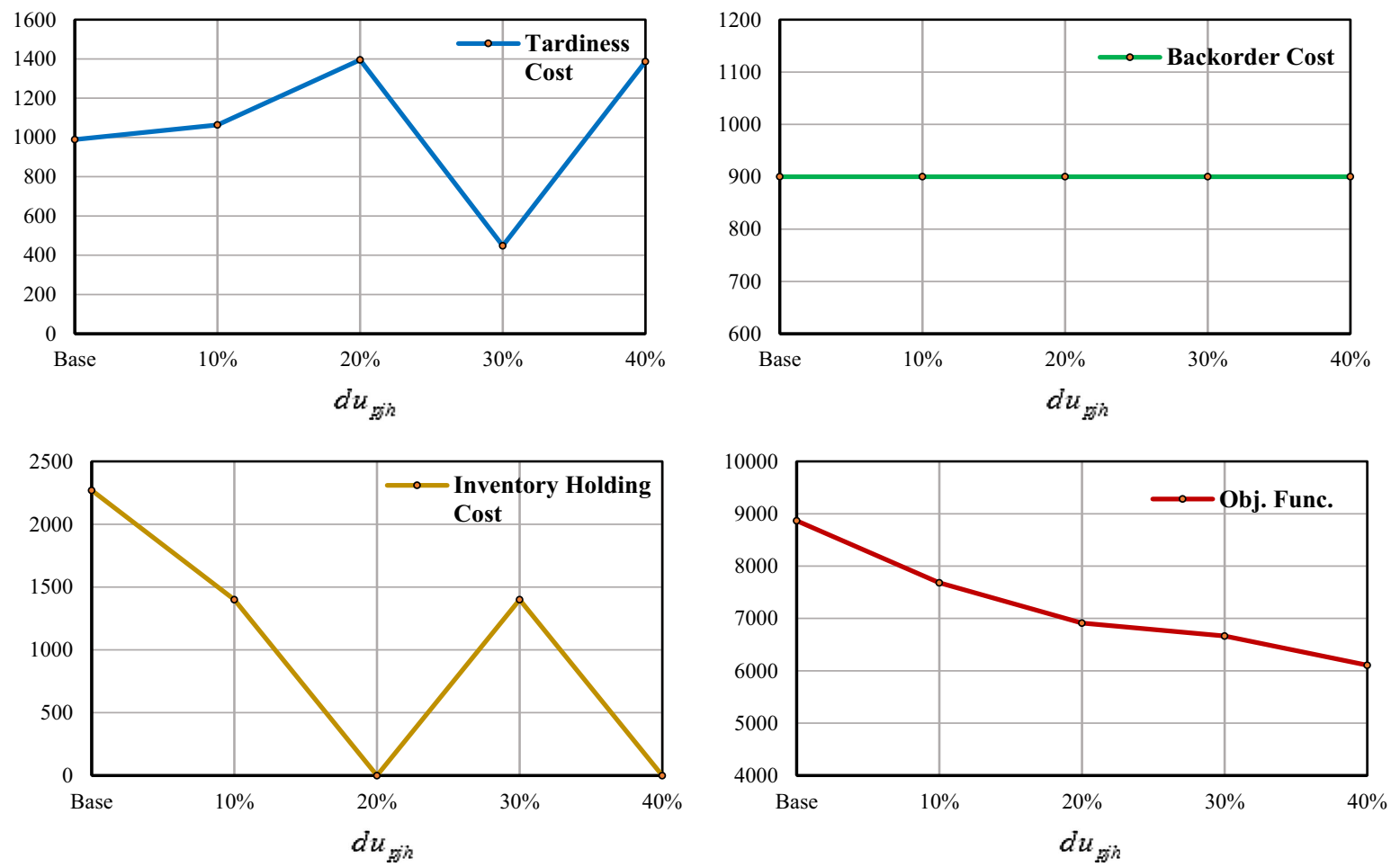

FiguRE 16. Influence of due date variations over the tardiness cost, backorder cost, inventory holding cost, and the objective function value.

periods (the tardiness or backorder costs becomes higher than inventory holding costs; thus, the model prefers to undergo the inventory holding costs); however, regarding this increase in due dates, the tardiness cost will be less than its previous amount. Thus, the model prefers to pay the pertinent tardiness cost and deliver the part in its related period.

The effect of lead time variation on the objective function components is depicted in Figure 17. Generally, we can speculate that by increasing the lead time, some raw materials are received after the appropriate time to start producing parts in the required period. As a result, PP strategies (e.g., backordering and inventory holding) will be effective to reduce the tardiness cost happening due to late receipt of raw materials. In brief, increment in the objective function by raising the lead time of raw materials is shown in Figure 17 as we expect that growth.

The effect of an increase in transportation time by vehicles on the objective function components is depicted in Figure 18. It is easily predictable that by increasing the transportation time from the plant to the markets, some parts may be delivered to the markets with some tardiness. As a result, a tardiness cost rises by increasing transportation time and the total objective function is affected in the same manner. The amount of growth in the objective function value due to increasing lead time proves the importance of the lead time parameter.

The effect of an increase in the raw materials order cost on the objective function components is predictable similar to increase in their lead time as depicted in Figure 19.

As seen in Figure 20, the trends which are observed by increasing the backorder and inventory holding costs on the objective function are similar to each other and predictable as well. This may also raise a concern as by increasing these parameters from 30 to $40 \%$, a reduction in the objective function value is seen that is against our prediction. The cause of this behavior of the model is that the CPLEX software is interrupted in 

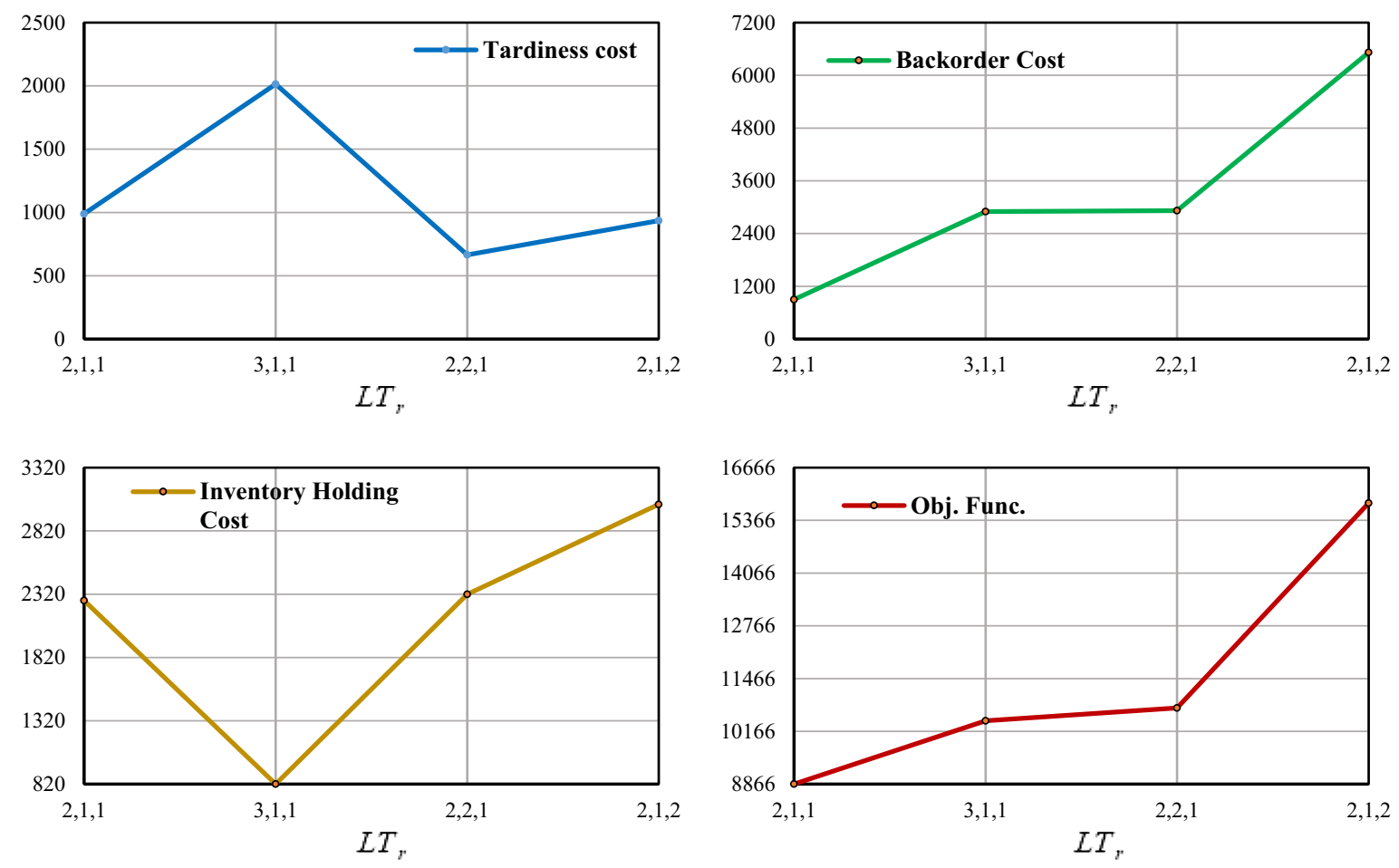

FiguRE 17. Influence of lead time variations over the tardiness cost, backorder cost, inventory holding cost, and the objective function value.

a predetermined time before obtaining an optimal solution. As a result, by changing input parameters (i.e., here are backorder and inventory holding costs), the more computational effort may be needed to obtain an optimal solution. Hence, the optimal objective function value in the level of $30 \%$ increases in the considered parameters should be lower than the current reported ones. This justifies unprecedented OFV in Figure 20.

Although increasing machines' relocation cost affects the OFV as we anticipate; however, its effect is marginal and shows that this parameter is not decisive on the solution of the deigned example.

As another example, increasing the intracellular and intercellular movement times resulted in some turbulence in the value of the objective function in some cases. The trends for OFV in Figure 22 are ascending as it is expected, there is one unprecedented climb in OFV for $10 \%$ increase in inter-cell movement cost tough. This happened due to interruption in a solution process of CPLEX manually and reporting best-known solution at that stage. So, the obtained solution is not an optimal one, which should lower than reported value at level $10 \%$. Nevertheless, it seems that the model is not very sensitive respect to these two parameters in solving the designed example.

Generally, there are clear explanations for all trends on the OFV as the input parameters are changed as well as some unexpected behaviors; however, obviously, tracking and expounding all those behaviors are out of the intention of this study.

\subsection{SA parameters tuning}

The efficiency of an algorithm is highly influenced by its parameters. Therefore, various levels of parameters may generate different solutions with diverse qualities. If the parameters are not set appropriately, it is not possible to find optimal or near-optimal solutions. 

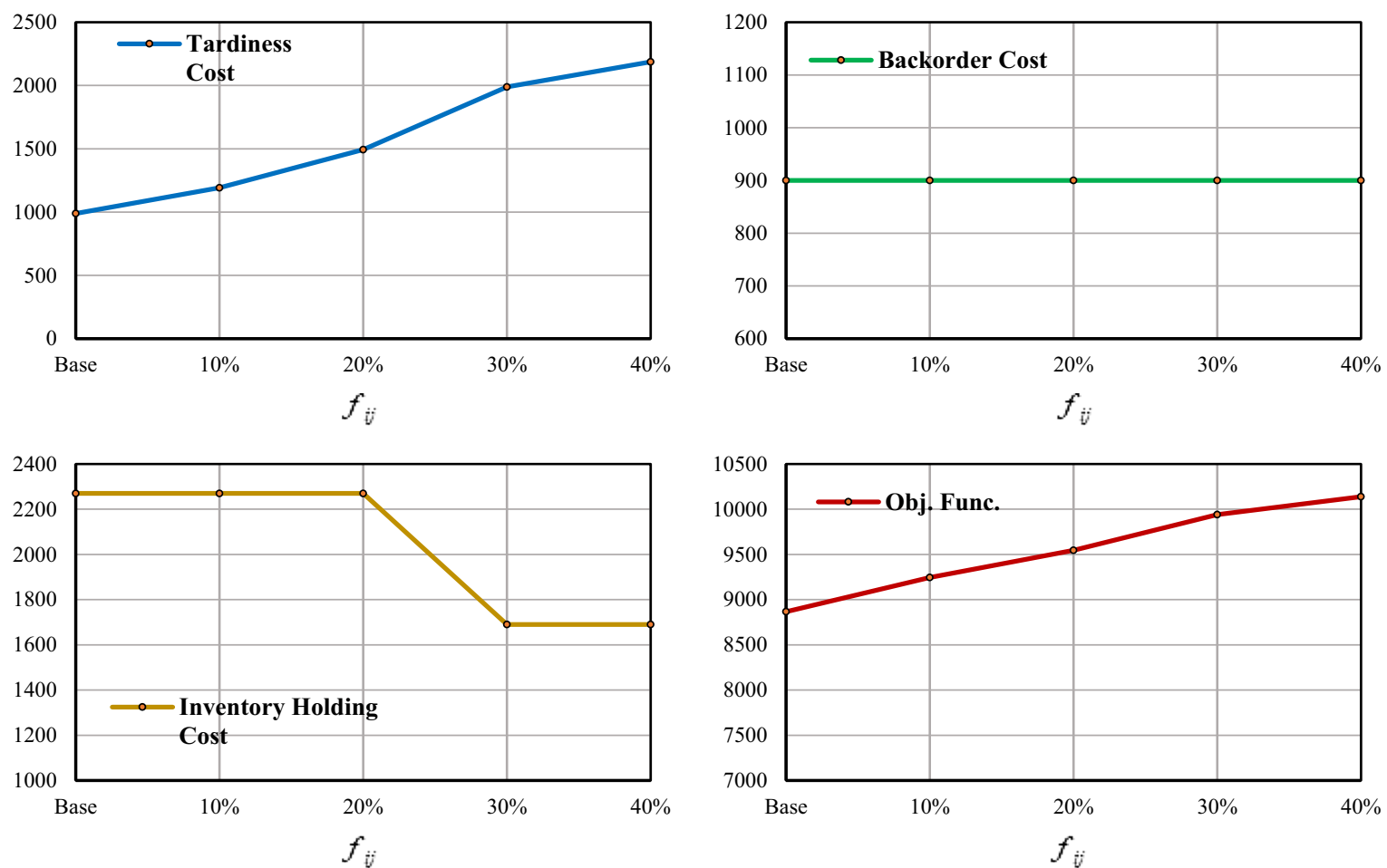

FIGURE 18. Influence of vehicles' required time variations over the tardiness cost, backorder cost, inventory holding cost, and the objective function value.

To tune the parameters of an algorithm, there are several methods to statistically design the experimental examination. However, the most commonly-used and exhaustive method is the full factorial experiment $[41,54]$ which is not always effective because of becoming increasingly difficult to perform examinations while the number of factors becomes considerably large. To decrease the number of required examinations, a fractional factorial experiment (FFE) method was introduced by Cochran and Cox [10]. Taguchi [57] developed a group of FFE matrices that eventually decreases the number of experimentations, although makes available sufficient information. Using a small number of experiments in the Taguchi method, orthogonal arrays are employed to investigate the effects of a large number of different combinations of parameters Taguchi technique is employed to optimally estimate various parameters affecting a model and it is based on mathematical computations and the design of experiments

In this technique, first off the parameters influencing the algorithm performance are identified and analyzed based on the input indices where usually the objective function is employed. Then, some combinations of the parameters' diverse levels are determined and the model is solved by the proposed algorithm with several iterations (here is 5). Based on the given solutions, the efficient values are calculated for the parameters setting.

In this study, the Taguchi technique has been applied by Minitab 17 to set the proposed SA parameters. To achieve better values of parameters for the problems in different sizes, the parameters are set for both small and large ones. Also, 4 parameters of SA algorithm and 4 levels for each one have been taken into account as seen in Table 13.

Figure 23 demonstrates the diagram for the mean signal to noise in the Taguchi method for small-sized problems. According to the final results, 2500 is chosen for $T_{\text {zero }}, 10$ for $T_{\text {final }}, 80$ for $N_{\text {var }}$ and 0.995 for $\alpha$. 

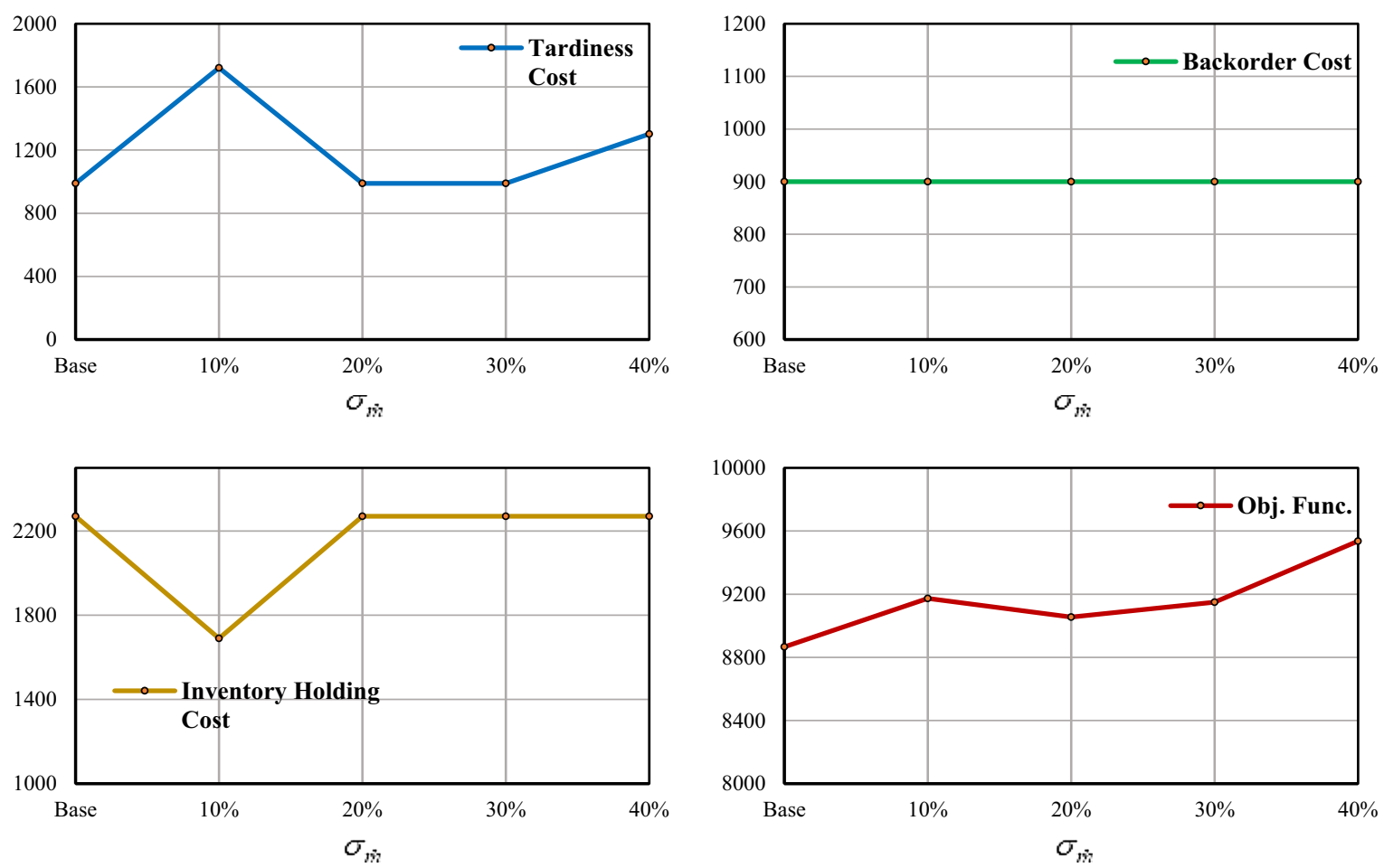

FiguRE 19. Influence of ordering cost variations over the tardiness cost, backorder cost, inventory holding cost, and the objective function value.
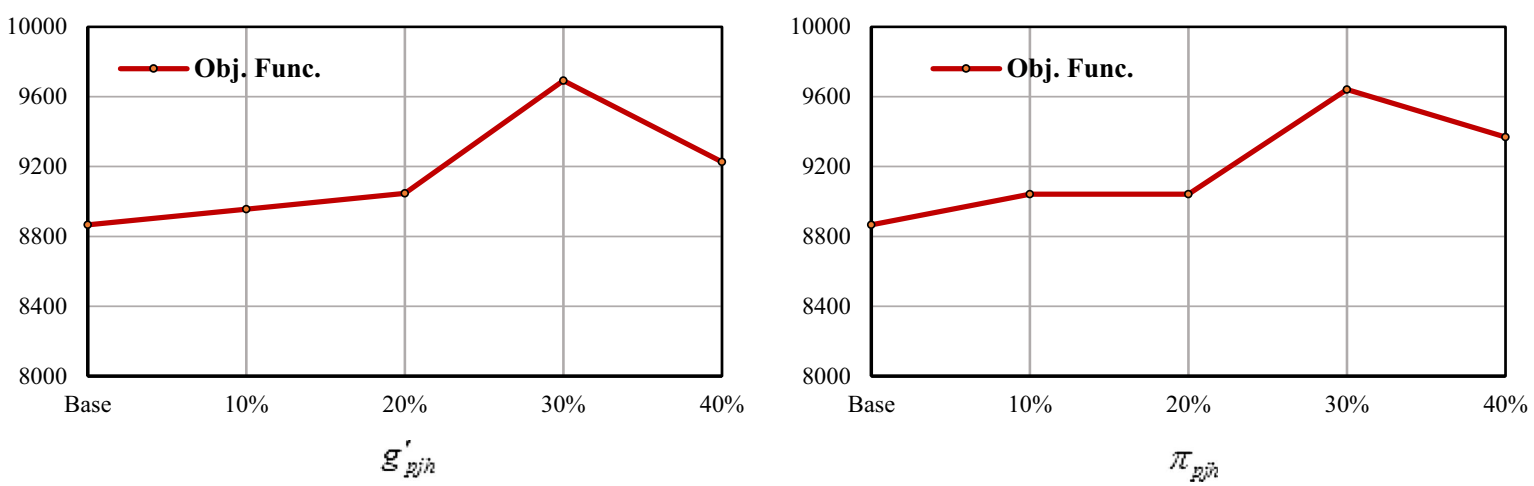

FigURE 20. Influence of backorder cost and inventory holding cost variations over the objective function value.

Similarly, Figure 24 demonstrates the diagram for the mean signal to noise in the Taguchi method for large-sized problems. According to the final results, 3000 is chosen for $T_{z e r o}, 5$ for $T_{\text {final }}, 120$ for $N_{\text {var }}$ and 0.99 for $\alpha$.

\subsection{Computational results}

To evaluate the performance of the developed SA in comparison with CPLEX, 20 numerical examples with various sizes either generated randomly or taken from the literature are solved. In the first 14 examples, 

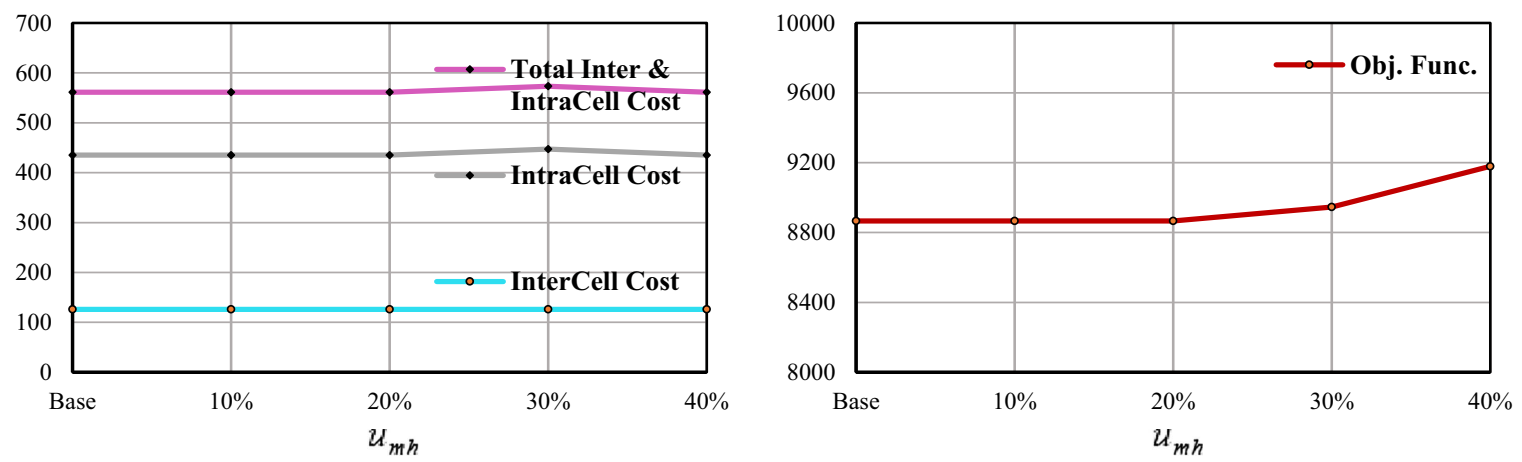

FIGURE 21. Influence of machines' relocation cost variations over the objective function value.


FiguRE 22. Influence of intra- and inter-cell movement cost variations over the objective function value.

TABLE 13. Considered levels for parameters of the SA algorithm.

\begin{tabular}{llllll}
\hline \hline Problem size & Parameters & Level 1 & Level 2 & Level 3 & Level 4 \\
\hline \multirow{4}{*}{ Small } & $T_{\text {zero }}$ & 1000 & 1500 & 2000 & 2500 \\
& $T_{\text {final }}$ & 10 & 15 & 20 & 25 \\
& $N_{\text {var }}$ & 80 & 100 & 120 & 140 \\
& $\alpha$ & 0.98 & 0.985 & 0.99 & 0.995 \\
Large & $T_{\text {zero }}$ & 1000 & 3000 & 5000 & 8000 \\
& $T_{\text {final }}$ & 5 & 10 & 15 & 20 \\
& $N_{\text {var }}$ & 80 & 100 & 120 & 140 \\
& $\alpha$ & 0.98 & 0.985 & 0.99 & 0.995 \\
\hline
\end{tabular}

the tuned parameters for small-sized problems and in the examples $15-20$, the tuned parameters for largesized problems have been employed.

The SA algorithm is coded in Visual Basic software and run 50 replicates on an Intel(R) core(TM) i5 CPU 2.6 GHz computer with $4.00 \mathrm{~GB}$ RAM for each test problem, and the best-obtained solution in 50 runs is reported in Table 14. 


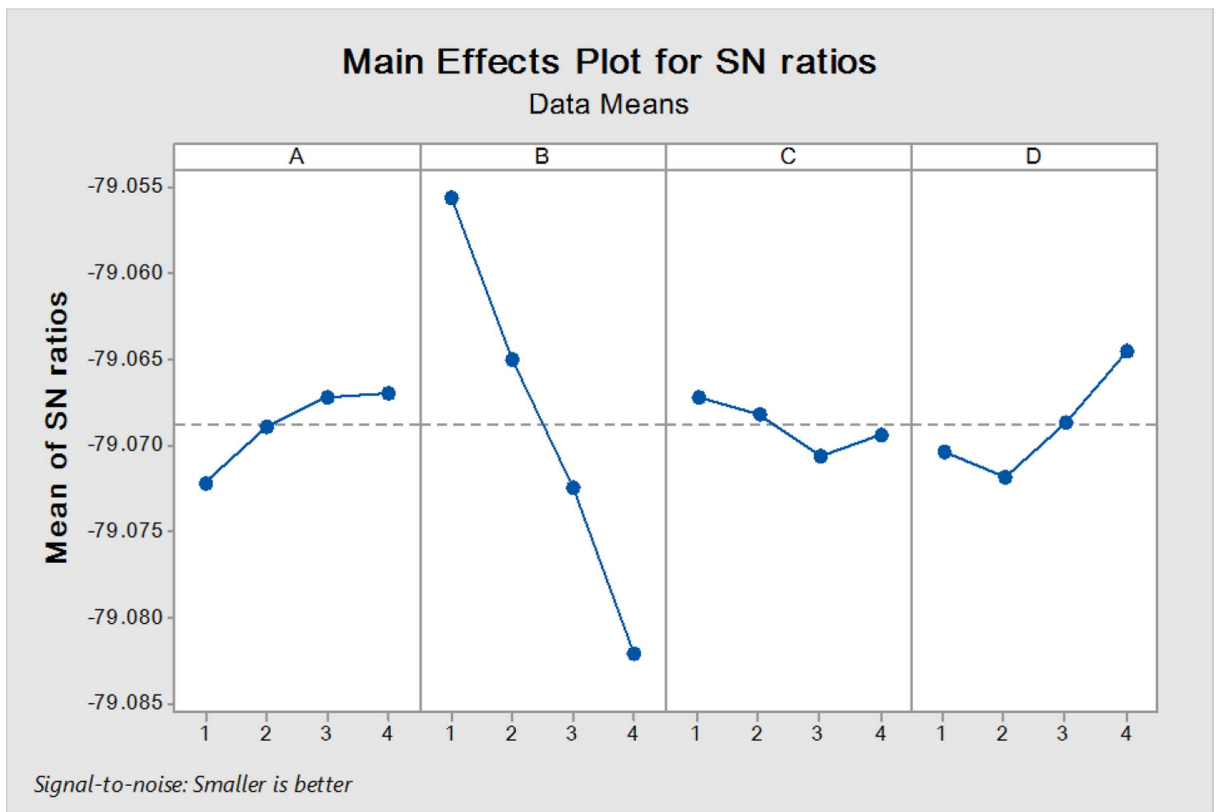

Figure 23. Main effects plot for the $\mathrm{S} / \mathrm{N}$ ratios for small-sized problems.

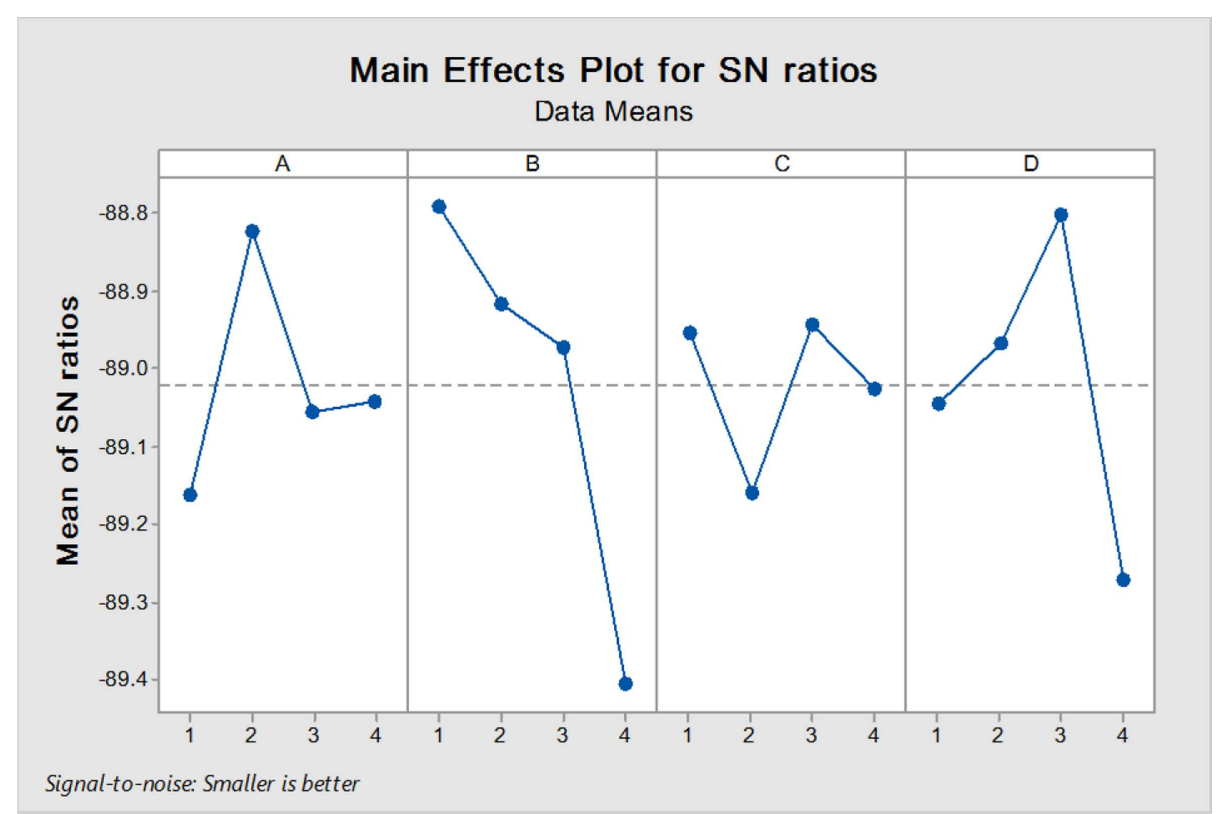

Figure 24. Main effects plot for the $\mathrm{S} / \mathrm{N}$ ratios for large-sized problems. 
Table 14 illustrates the information of test problems and the Objective Function Values (OFV) along with the computational times obtained by the SA algorithm and CPLEX solver. As can be seen from Table 14, SA has obtained the optimal solutions for problems 3, 5, 6 and 10. Furthermore, SA has found near-optimal solutions with an average gap less than 8 percent in much less computational times than CPLEX for problems 1, 2, 4, 7, 8 and 11. Besides, in large-sized problems 9 and 10, CPLEX solver has been interrupted before encountering "Out of Memory" error and the best-obtained solution has been reported. In other test problems including 12-20 even no feasible solution is found before encountering out of memory error due to an extremely high level of computational complexity and as a result, no solution is reported. It has to be noted that the notation "** in Table 14 stands for "Out of Memory" message shown in GAMS software.

These satisfactory results obtained by the proposed SA prove the efficiency of the designed algorithm enhanced by the matrix-based chromosome structure and hierarchical solution generation procedure in solving the proposed model.

\section{Conclusion}

The design and operation of production systems in the current era of global competition are becoming a difficult task and very complex. Modeling and optimization of such complex systems are of paramount importance in achieving competitive advantages. This paper has presented an MINLP model integrating the design of a CMS in a supply chain with PP, TP and parts scheduling decisions under a dynamic environment with a solution approach based on a SA algorithm. In summary, contributions of this model could be summarized as follows:

A three-layer supply chain encompassing the raw materials supplier, the manufacturing plant and the markets have been investigated. At the 1st layer, determining the time and quantities of the raw materials orders are dealt with. At the 2nd layer, the configuration of the cells, the parts operations assignment to the cells and the scheduling of the parts inside the plant are addressed. And ultimately, at the 3rd level, via selecting the suitable vehicles, the parts are delivered to the markets.

The presented model has integrated a huge portion of design features, such as considering a supply chain in a three-layer network with multi-markets, multi-period production planning, parts due date, cell reconfiguration, time and cost of intracellular/intercellular movement, parts scheduling, alternative processing routes, operation sequence, lead time and usage coefficient of raw materials, machine capacity, manufacturing time, cell size limit and transportation time. The extended model was capable of determining optimally: (1) scheduling decisions including the amount of tardiness for each part, the start time and ending time of manufacturing each part operation, and the completion time of each part, (2) PP decisions including the production periods for each part, order amounts of raw materials, inventory level of raw materials and parts, and backorders volume, (3) the intracellular/intercellular movements, (4) the cell reconfigurations, and (5) transportation plan of parts from the plant to the markets. The objective was to minimize total costs of tardiness, intracellular/intercellular movement, machines relocation, purchase and inventory holding of raw materials, parts transportation from the plant to the markets, backorders and parts inventory holding.

To demonstrate the model distinctive capabilities, an illustrative numerical example is solved using CPLEX solver with several sensitivity analyses. The obtained solutions have also demonstrated that this model, to an extent, conquers common disadvantages existing in the presented DCMS models, which have not been yet integrated with other interrelated problems such as PP, TP and parts scheduling simultaneously. However, such a comprehensive model is difficult to solve using off-the-shelf optimization packages employing exact methods such as a branch and cut algorithm. To this end, in this paper, we developed an efficient SA meta-heuristic algorithm for large dimension problems. Moreover, to tune the proposed algorithm parameters, Taguchi technique has been employed. Finally, the computational results revealed that the developed SA had a satisfactory performance in generating near-optimal solutions in comparison with CPLEX solver based on the objective function values and computational time Besides, for future studies, we can point out the following cases: employing the other new meta-heuristic algorithms, taking into account the layout of machines and cells, designing multi-objective 


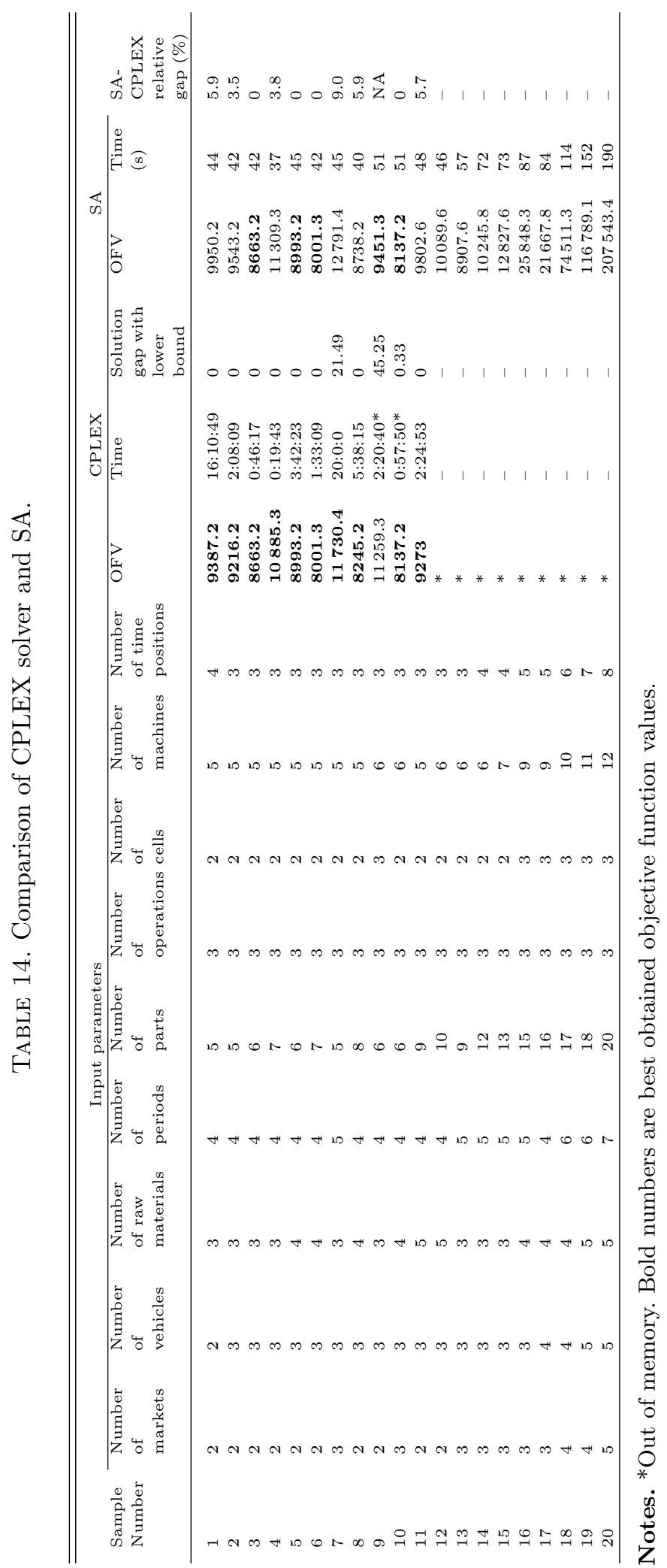


models for the problem, investigating the effects of learning and forgetting on the production system, considering assignment of workers with multi-level flexibility, machine reliability issues, and considering uncertainty for parts demands, machine capacity and cost parameters. Another possible extension to this research can be empowering the proposed SA with embedding a linear program sub-problem in the stochastic search of the SA algorithm as the applicability of the proposed SA is limited in solving a real case with large sizes.

\section{REFERENCES}

[1] A. Aalaei and H. Davoudpour, Revised multi-choice goal programming for incorporated dynamic virtual cellular manufacturing into supply chain management: a case study. Eng. App. Artif. Intell. 47 (2016) 3-15.

[2] A. Aghajani, S.A. Didehbani, M. Kazemi and N. Javadian, A dynamic non-linear mixed integer-programming model for the CMS design with production planning. Int. J. Ind. Syst. Eng. 16 (2014) 70-87.

[3] S. Ahkioon, A.-A. Bulgak and T. Bektas, Integrated cellular manufacturing systems design with production planning and dynamic system reconfiguration. Eur. J. Oper. Res. 192 (2009) 414-428.

[4] A. Alfieri and G. Nicosia, Sequencing a batching flexible cell to minimise set-up costs. Int. J. Prod. Res. 52 (2014) $2461-2476$.

[5] F. Alhourani, Cellular manufacturing system design considering machines reliability and parts alternative process routings. Int. J. Prod. Res. 54 (2016) 846-863.

[6] J. Arkat, M.H. Farahani and L. Hosseini, Integrating cell formation with cellular layout and operations scheduling. Int. J. Adv. Manuf. Technol. 61 (2012) 637-647.

[7] A. Azadeh, M. Ravanbakhsh, M. Rezaei-Malek, M. Sheikhalishahi and A. Taheri-Moghaddam, Unique NSGA-II and MOPSO algorithms for improved dynamic cellular manufacturing systems considering human factors. Appl. Math. Model. 48 (2017) 655-672.

[8] H. Bayram and R. Şahin, A comprehensive mathematical model for dynamic cellular manufacturing system design and Linear Programming embedded hybrid solution techniques. Comput. Ind. Eng. 91 (2016) 10-29.

[9] J.L. Burbidge, Production flow analysis. Prod. Eng. 50 (1971) 139-152.

[10] W.G. Cochran and G.M. Cox, Experimental Designs, 2nd edtion. John Wiley \& Sons, New York, NY (1992).

[11] K. Deep and P.K. Singh, Design of robust cellular manufacturing system for dynamic part population considering multiple processing routes using genetic algorithm. J. Manuf. Syst. 35 (2015) 155-163.

[12] F.M. Defersha and A. Hodiya, A mathematical model and a parallel multiple search path simulated annealing for an integrated distributed layout design and machine cell formation. J. Manuf. Syst. 43 (2017) 195-212.

[13] A. Delgoshaei and G. Chandima, 2016. A multi-layer perceptron for scheduling cellular manufacturing systems in the presence of unreliable machines and uncertain cost. Appl. Soft Comput. 49 (2016) 27-55.

[14] A. Delgoshaei, A. Ali, M.K.A. Ariffin and C. Gomes, A multi-period scheduling of dynamic cellular manufacturing systems in the presence of cost uncertainty. Comput. Ind. Eng. 100 (2016) 110-132.

[15] J. Drolet, Y. Marcoux and G. Abdulnour, Simulation-based performance comparison between dynamic cells, classical cells and job shops: a case study. Int. J. Prod. Res. 46 (2008) 509-536.

[16] A. Ebrahimi, R. Kia and A.R. Komijan, Solving a mathematical model integrating unequal-area facilities layout and part scheduling in a cellular manufacturing system by a genetic algorithm. SpringerPlus $\mathbf{5}$ (2016) 1254.

[17] H. Feng, L. Xi, T. Xia and E. Pan, Concurrent cell formation and layout design based on hybrid approaches. Appl. Soft Comput. 66 (2018) 346-359.

[18] V.R. Ghezavati, A new stochastic mixed integer programming to design integrated cellular manufacturing system: a supply chain framework. Int. J. Ind. Eng. Comput. 2 (2011) 563-574.

[19] V.R. Ghezavati, Designing integrated cellular manufacturing systems with tactical decisions. J. Chin. Inst. Eng. 38 (2015) 332-341.

[20] V.R. Ghezavati, S. Sadjadi and M. Dehghan Nayeri, Integrating strategic and tactical decisions to robust designing of cellular manufacturing under uncertainty: fixed suppliers in supply chain. Int. J. Comput. Intel. Syst. 4 (2011) 837-854.

[21] T. Ghosh, B. Doloi and P.K. Dan, Applying soft-computing techniques in solving dynamic multi-objective layout problems in cellular manufacturing system. Int. J. Adv. Manuf. Technol. 86 (2016) 237-257.

[22] K. Halat and R. Bashirzadeh, Concurrent scheduling of manufacturing cells considering sequence-dependent family setup times and intercellular transportation times. Int. J. Adv. Manuf. Technol. 77 (2015) 1907-1915.

[23] S.S. Heragu, Group technology and cellular manufacturing. IEEE Trans. Syst. Man Cybern. 24 (1994) $203-214$.

[24] A. Iqbal and K.A. Al-Ghamdi, Energy-efficient cellular manufacturing system: eco-friendly revamping of machine shop configuration. Energy 163 (2018) 863-872.

[25] F. Khaksar-Haghani, R. Kia, N. Javadian, R. Tavakkoli-Moghaddam and A. Baboli, A comprehensive mathematical model for the design of a dynamic cellular manufacturing system integrated with production planning and several manufacturing attributes. Int. J. Ind. Eng. Prod. Res. 22 (2011) 199-212.

[26] R. Kia, A. Baboli, N. Javadian, R. Tavakkoli-Moghaddam, M. Kazemi and J. Khorrami, Solving a group layout design model of a dynamic cellular manufacturing system with alternative process routings, lot splitting and flexible reconfiguration by simulated annealing. Comput. Oper. Res. 39 (2012) 2642-2658. 
[27] R. Kia, N. Javadian, M.M. Paydar and M. Saidi-Mehrabad, A simulated annealing for intra-cellularlayout design of dynamic cellular manufacturing systems with route selection, purchasing machines and cell reconfiguration. Asia-Pac. J. Oper. Res. 30 (2013) 1350004.

[28] R. Kia, N. Javadian and R. Tavakkoli-Moghaddam, A simulated annealing algorithm to determine a group layout and production plan in a dynamic cellular manufacturing system. J. Optim. Ind. Eng. 14 (2014) 37-52.

[29] R. Kia, M. Kazemi, S. Shafiee Gol, R. Tavakkoli-Moghaddam and J. Khorrami, A mathematical model for assessing the effects of a lot splitting feature on a dynamic cellular manufacturing system. Prod. Eng. 11 (2017) 557-573.

[30] S. Kirkpatrick, C.D. Gelatt and M.P. Vecchi, Optimization by simulated annealing. Science 220 (1983) $671-680$.

[31] J. Li, A. Wang and C. Tang, Production planning in virtual cell of reconfiguration manufacturing system using genetic algorithm. Int. J. Adv. Manuf. Technol. 74 (2014) 47-64.

[32] Y. Li, X. Li and J.N. Gupta, Solving the multi-objective flowline manufacturing cell scheduling problem by hybrid harmony search. Expert Syst. App. 42 (2015) 1409-1417.

[33] C. Liu and J. Wang, Cell formation and task scheduling considering multi-functional resource and part movement using hybrid simulated annealing. Int. J. Comput. Intell. Syst. 9 (2016) $765-777$.

[34] C. Liu, J. Wang and J.Y.T. Leung, Worker assignment and production planning with learning and forgetting in manufacturing cells by hybrid bacteria foraging algorithm. Comput. Ind. Eng. 96 (2016) 162-179.

[35] C. Liu, J. Wang and J.Y.T. Leung, Integrated bacteria foraging algorithm for cellular manufacturing in supply chain considering facility transfer and production planning. Appl. Soft Comput. 62 (2018) 602-618.

[36] K.S. Lokesh and P.K. Jain, An integrated model of dynamic cellular manufacturing and supply chain system design. Int. J. Adv. Manuf. Technol. 62 (2012) 385-404.

[37] E. Mehdizadeh and V. Rahimi, An integrated mathematical model for solving dynamic cell formation problem considering operator assignment and inter/intra cell layouts. Appl. Soft Comput. 42 (2016) 325-341.

[38] S.P. Mitrofanov, The Scientific Principles of Group Technology. National Lending Library Translation, Boston, MA (1966).

[39] M. Mohammadi and K. Forghani, Designing cellular manufacturing systems considering S-shaped layout. Comput. Ind. Eng. 98 (2016) 221-236.

[40] S. Molla-Alizadeh-Zavardehi, S. Sadi Nezhad, R. Tavakkoli-Moghaddam and M. Yazdani, Solving a fuzzy fixed charge solid transportation problem by metaheuristics. Math. Comput. Model. 57 (2013) 1543-1558.

[41] D.C. Montgomery, Design and Analysis of Experiments. John Wiley \& Sons, New York, NY (2017).

[42] F. Niakan, A. Baboli, T. Moyaux and V. Botta-Genoulaz, A bi-objective model in sustainable dynamic cell formation problem with skill-based worker assignment. J. Manuf. Syst. 38 (2016) 46-62.

[43] J. Olhager, Evolution of operations planning and control: from production to supply chains. Int. J. Prod. Res. 51 (2013) 6836-6843.

[44] M. Pajoutan, A. Golmohammadi and M. Seifbarghy, CMS scheduling problem considering material handling and routing flexibility. Int. J. Adv. Manuf. Technol. 72 (2014) 881-893.

[45] M.M. Paydar and M. Saidi-Mehrabad, Revised multi-choice goal programming for integrated supply chain design and dynamic virtual cell formation with fuzzy parameters. Int. J. Comput. Integr. Manuf. 28 (2015) 251-265.

[46] M.M. Paydar, M. Saidi-Mehrabad and E. Teimoury, A robust optimisation model for generalised cell formation problem considering machine layout and supplier selection. Int. J. Comput. Integr. Manuf. 27 (2014) 772-786.

[47] A. Pailla, A.R. Trindade, V. Parada and L.S. Ochi, A numerical comparison between simulated annealing and evolutionary approaches to the cell formation problem. Expert Syst. App. 37 (2010) 5476-5483.

[48] K. Rafiee, M. Rabbani, H. Rafiei and A. Rahimi-Vahed, A new approach towards integrated cell formation and inventory lot sizing in an unreliable cellular manufacturing system. Appl. Math. Modell. 35 (2011) 1810-1819.

[49] P.P. Rao and R. Mohanty, Impact of cellular manufacturing on supply chain management: exploration of interrelationships between design issues. Int. J. Manuf. Technol. Manage. 5 (2003) 507-520.

[50] H. Raoofpanah, V. Ghezavati and R. Tavakkoli-Moghaddam, Solving a new robust green cellular manufacturing problem with environmental issues under uncertainty using Benders decomposition. Eng. Optim. 51 (2019) 1229-1250.

[51] H. Rafiei, M. Rabbani, H. Gholizadeh and H. Dashti, A novel hybrid SA/GA algorithm for solving an integrated cell formationjob scheduling problem with sequence-dependent set-up times. Int. J. Manage. Sci. Eng. Manage. 11 (2016) 134-142.

[52] P. Renna and M. Ambrico, Design and reconfiguration models for dynamic cellular manufacturing to handle market changes. Int. J. Comput. Integr. Manuf. 28 (2015) 170-186.

[53] M. Rheault, J. Drolet and G. Abdulnour, Physically reconfigurable virtual cells: a dynamic model for a highly dynamic environment. Comput. Ind. Eng. 29 (1995) 221-225.

[54] R. Ruiz, C. Maroto and J. Alcaraz, Solving the flowshop scheduling problem with sequence dependent setup times using advanced metaheuristics. Eur. J. Oper. Res. 165 (2005) 34-54.

[55] M. Sakhaii, R. Tavakkoli-Moghaddam, M. Bagheri and B. Vatani, A robust optimization approach for an integrated dynamic cellular manufacturing system and production planning with unreliable machines. Appl. Math. Modell. 40 (2016) 169-191.

[56] F. Shafigh, F.M. Defersha and S.E. Moussa, A linear programming embedded simulated annealing in the design of distributed layout with production planning and systems reconfiguration. Int. J. Adv. Manuf. Technol. 88 (2017) 1119-1140.

[57] G. Taguchi, Introduction to quality engineering: designing quality into products and processes. White Plains: Asian Productivity Organisation/UNIPUB, Tokyo (1986).

[58] J. Tang, X. Wang, I. Kaku and K.L. Yung, Optimization of parts scheduling in multiple cells consideringintercell move using scatter search approach. J. Intell. Manuf. 21 (2010) 525-537. 
[59] J. Tang, C. Yan, X. Wang and C. Zeng, Using Lagrangian relaxation decomposition with heuristic to integrate the decisions of cell formation and parts scheduling considering intercell moves. IEEE Trans. Autom. Sci. Eng. 11 (2014) 1110-1121.

[60] U. Wemmerlov and N.L. Hyer, Procedures for the part family/machine group identification problem in cellular manufacturing. J. Oper. Manage. 6 (1986) 125-147.

[61] U. Wemmerlov and N.L. Hyer, Cellular Manufacturing in the US industry: a survey of users. Int. J. Prod. Res. 27 (1989) $1511-1530$.

[62] X. Wu, C.H. Chu, Y. Wang and D. Yue, Genetic algorithms for integrating cell formation with machine layout and scheduling. Comput. Ind. Eng. 53 (2007) 277-289.

[63] J. Wang, C. Liu and K. Li, A hybrid simulated annealing for scheduling in dual-resource cellular manufacturing system considering worker movement. Automatika 60 (2019) 172-180.

[64] A. Zeb, M. Khan, N. Khan, A. Tariq, L. Ali, F. Azam and S.H.I. Jaffery, Hybridization of simulated annealing with genetic algorithm for cell formation problem. Int. J. Adv. Manuf. Technol. 86 (2016) 2243-2254. 